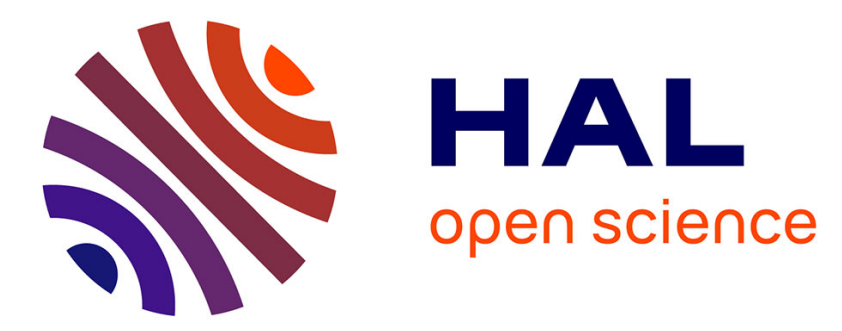

\title{
Asynchronous Template Games and the Gray Tensor Product of 2-Categories
}

\author{
Paul-André Melliès
}

\section{To cite this version:}

Paul-André Melliès. Asynchronous Template Games and the Gray Tensor Product of 2-Categories. LICS 2021 - 36th Annual ACM/IEEE Symposium on Logic in Computer Science, Jul 2021, Rome, Italy. 10.1109/LICS52264.2021.9470758 . hal-03455968

\section{HAL Id: hal-03455968 \\ https://hal.inria.fr/hal-03455968}

Submitted on 29 Nov 2021

HAL is a multi-disciplinary open access archive for the deposit and dissemination of scientific research documents, whether they are published or not. The documents may come from teaching and research institutions in France or abroad, or from public or private research centers.
L'archive ouverte pluridisciplinaire HAL, est destinée au dépôt et à la diffusion de documents scientifiques de niveau recherche, publiés ou non, émanant des établissements d'enseignement et de recherche français ou étrangers, des laboratoires publics ou privés. 


\title{
Asynchronous Template Games and the Gray Tensor Product of 2-Categories
}

\author{
Paul-André Melliès \\ Institut de Recherche en Informatique Fondamentale (IRIF) \\ CNRS, Université de Paris, France \\ Email: mellies@irif.fr
}

\begin{abstract}
In his recent and exploratory work on template games and linear logic, Melliès defines sequential and concurrent games as categories with positions as objects and trajectories as morphisms, labelled by a specific synchronization template. In the present paper, we bring the idea one dimension higher and advocate that template games should not be just defined as 1-dimensional categories but as 2-dimensional categories of positions, trajectories and reshufflings (or reschedulings) as 2cells. In order to achieve the purpose, we take seriously the parallel between asynchrony in concurrency and the Gray tensor product of 2-categories. One technical difficulty on the way is that the category $\mathbb{S}=\mathbf{2}$-Cat of small 2-categories equipped with the Gray tensor product is monoidal, and not cartesian. This prompts us to extend the framework of template games originally formulated by Melliès in a category $\mathbb{S}$ with finite limits, and to upgrade it in the style of Aguiar's work on quantum groups to the more general situation of a monoidal category $\$$ with coreflexive equalizers, preserved by the tensor product componentwise. We construct in this way an asynchronous template game semantics of multiplicative additive linear logic (MALL) where every formula and every proof is interpreted as a labelled 2-category equipped, respectively, with the structure of Gray comonoid for asynchronous template games, and of Gray bicomodule for asynchronous strategies.
\end{abstract}

\section{INTRODUCTION AND OVERVIEW}

Game semantics is the offspring of a fruitful encounter between concurrency theory, proof theory and programming language semantics. Arising at this crossover position between the three fields, game semantics inherits the rich toolbox of ideas and techniques of concurrency theory, and provides a vivid landscape of logical and programming phenomena to dissect and to interpret. For that reason, it was understood at an early stage of game semantics [1], [19] that this nice hybridation at the heart of game semantics would offer a fresh start and a number of new perspectives on the mathematical foundations of concurrency theory. Since the very origins of the field and the seminal contributions by Petri [26], [27], Bekič [5] and Mazurkiewicz [20], one main ambition of concurrency theory has been to give an abstract and mathematically elegant account of the symbolic choreography at work in a distributed (software or hardware) system of intercommunicating components.

Labelled transition systems: The description is typically based on the notion of transition system

$$
\text { Tran }=(G, \lambda)
$$

on a specific set $\mathscr{L}$ of labels, defined as a graph $G=$ $(V, E$, source, target) consisting of a set $V$ of vertices, a set $E$ of edges, and two functions source, target $: E \longrightarrow V$ equipped with a labelling function $\lambda: E \longrightarrow \mathscr{L}$. In this formulation, each state of the distributed system is represented as a vertex $x, y \in V$ and every transition $u: x \rightarrow y$ is represented as an edge $u \in E$ labelled by an element $\lambda(u) \in \mathscr{L}$. One sometimes requires in the traditional definition that there exists at most one transition $u: x \rightarrow y$ with a given label $\ell \in \mathscr{L}$ between two given states $x, y$; one thus writes $u=(x, \ell, y)$ for the unique edge $u: x \rightarrow y$ with label $\lambda(u)=\ell$. We do not make this simplifying assumption here, see [18] for a discussion.

Asynchronous transition systems: In order to capture the concurrent nature of computations, one equips the transition system Tran with a notion of independence between transitions $u$ and $v$ performed in separate components of the distributed system, with no shared memory. Because the two transitions $u$ and $v$ are independent, their order of execution does not matter. This idea is nicely captured mathematically by an intuition coming from algebraic topology and the connection between higher automata and cubical sets [14], [12]. A square in a graph $G$ is defined as a pair $(p, q)$ of paths $p, q: x \rightarrow y$ of length 2 , with same source $x$ and same target $y$, and thus of the form $p=u_{1} \cdot u_{2}$ and $q=v_{1} \cdot v_{2}$ as depicted below:

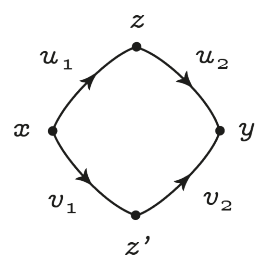

An asynchronous graph $(G, \diamond)$ is defined as a graph $G$ equipped with a set $\diamond$ of squares of $G$, satisfying three basic properties detailed in $\$$ III. We use the notation $p \diamond q$ when the square $(p, q)$ is an element of $\diamond$ and say in that case that the square $(p, q)$ defines a permutation square. Following an intuition coming from algebraic topology, a permutation tile $u_{1} \cdot u_{2} \diamond v_{1} \cdot v_{2}$ between the paths $p=u_{1} \cdot u_{2}$ and $q=v_{1} \cdot v_{2}$ is depicted in the following way, as a 2-dimensional surface (or tile) between the paths $p$ and $q$ :

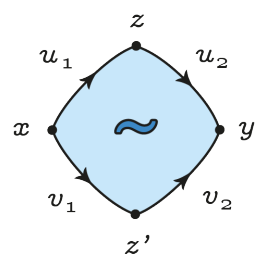


An asynchronous graph homomorphism

$$
f \quad: \quad\left(G, \diamond_{G}\right) \longrightarrow\left(H, \diamond_{H}\right)
$$

is defined as a graph homomorphism $f: G \rightarrow H$ with the additional property that every permutation square in $G$ is transported to a permutation square in $H$, in the sense that for every square $(p, q)$ in $G$,

$$
p \diamond_{G} q \Rightarrow f(p) \diamond_{H} f(q)
$$

where $f(p)=f\left(u_{1}\right) \cdot f\left(u_{2}\right)$ and $f(q)=f\left(v_{1}\right) \cdot f\left(v_{2}\right)$ are the paths of length 2 in the graph $H$ obtained as image of the paths $p=u_{1} \cdot u_{2}$ and $q=v_{1} \cdot v_{2}$. This defines the category Asynch of asynchronous graphs and asynchronous graph homomorphisms between them.

One nice and concise way to define a (labelled) asynchronous transition system from there is to associate to every set $\mathscr{L}$ the asynchronous graph noted

$$
\star[\mathscr{L}]=(\star[\mathscr{L}], \diamond \mathscr{L})
$$

with a unique vertex noted $*$, one edge $\ell: * \rightarrow *$ for each label $\ell \in \mathscr{L}$, and one permutation square

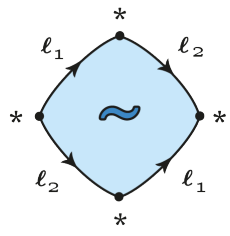

for every pair $\left(\ell_{1}, \ell_{2}\right)$ of (possibly equal) labels $\ell_{1}, \ell_{2} \in \mathscr{L}$. An asynchronous transition system is then defined as an asynchronous graph $(G, \diamond)$ equipped with an asynchronous graph homomorphism

$$
\lambda:(G, \diamond) \longrightarrow \star[\mathscr{L}]
$$

Note that the definition ensures that the labelling is asynchronous in the sense that $\lambda\left(u_{1}\right)=\lambda\left(v_{2}\right)$ and $\lambda\left(u_{2}\right)=\lambda\left(v_{1}\right)$ in any permutation square (4) of the asynchronous transition system $(G, \diamond)$. As we will see, this formulation (5) of asynchronous transition systems as a labelling map in the category Asynch is particularly convenient.

The shuffle tensor product: An important observation for this paper is that the category Asynch comes equipped with a symmetric monoidal structure, provided by the shuffle tensor product

$$
\left(G, \diamond_{G}\right) ш\left(H, \diamond_{H}\right) \quad=\quad\left(G \amalg H, \diamond_{G ш H}\right)
$$

of two asynchronous graphs $\left(G, \diamond_{G}\right)$ and $\left(H, \diamond_{H}\right)$. Here, $G \amalg H$ denotes the graph whose vertices $(x, y)$ are the pairs of a vertex $x$ in $G$ and of a vertex $y$ in $H$ and whose edges

$$
(x, y) \stackrel{(u, y)}{\longrightarrow}\left(x^{\prime}, y\right) \quad(x, y) \stackrel{(x, v)}{\longrightarrow}\left(x, y^{\prime}\right)
$$

are either pairs $(u, y)$ consisting of an edge $u: x \rightarrow x^{\prime}$ in $G$ and of a vertex $y$ in $H$; or symmetrically, pairs $(x, v)$ consisting of a vertex $x$ in $G$ and of an edge $v: y \rightarrow y^{\prime}$ in $H$. There is a permutation tile of $G \amalg H$

$$
(u, y) \cdot\left(x^{\prime}, v\right) \diamond_{G \amalg H}(x, v) \cdot\left(u, y^{\prime}\right)
$$

and in the reverse direction

$$
(x, v) \cdot\left(u, y^{\prime}\right) \diamond_{G \amalg H}(u, y) \cdot\left(x^{\prime}, v\right)
$$

as depicted below in orange
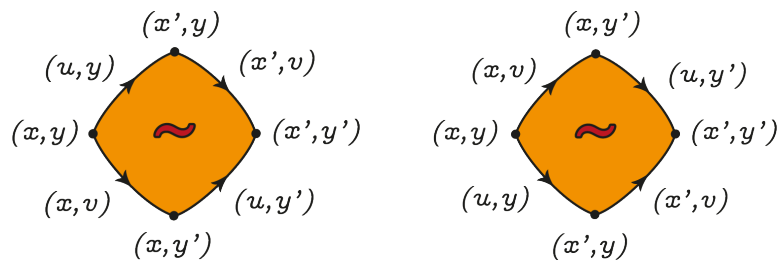

for every pair of edges $u: x \rightarrow x^{\prime}$ in $G$ and $v: y \rightarrow y^{\prime}$ in $H$; a permutation tile (in orange on the right-hand side)

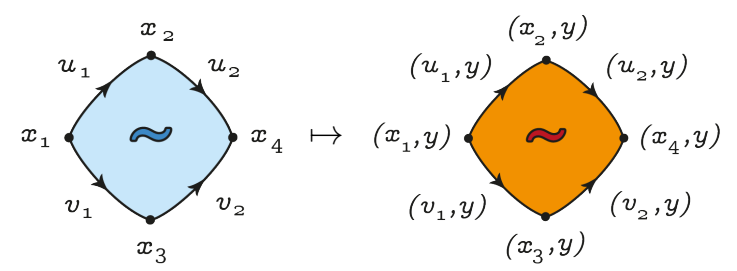

for every permutation tile $u_{1} \cdot u_{2} \diamond_{G} v_{1} \cdot v_{2}$ in $G$ (in blue on the left-hand side) and every vertex $y$ in $H$; symmetrically, a permutation tile (in orange on the right-hand side)

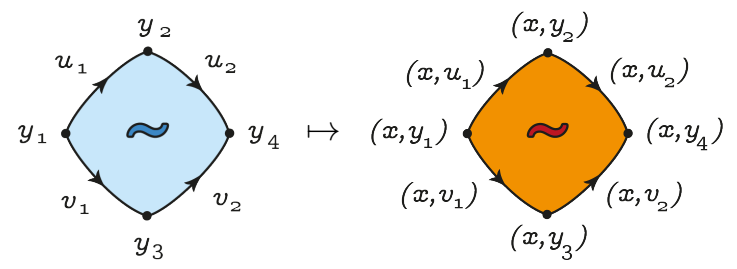

for every vertex $x$ in $G$ and permutation tile $u_{1} \cdot u_{2} \diamond_{G} v_{1} \cdot v_{2}$ in $H$ (in blue on the left-hand side). The unit of the shuffle tensor product is the asynchronous graph $I$ with one vertex and no edge. One establishes that

Proposition 1: The category Asynch of asynchronous graphs equipped with the shuffle tensor product $\amalg$ and the unit $I$ defines a symmetric monoidal category.

An additional observation is that the construction $t[-]$ described in (3) defines a symmetric monoidal functor

$$
\star[-]:(\text { Set },+, \varnothing) \longrightarrow(\text { Asynch, },, I)
$$

with monoidal coercions given by the expected isomorphisms:

$$
\begin{array}{cccc}
m_{\mathscr{L}_{1}, \mathscr{L}_{2}}: & \star\left[\mathscr{L}_{1}\right] \uplus \star\left[\mathscr{L}_{2}\right] & \longrightarrow & \star\left[\mathscr{L}_{1}+\mathscr{L}_{2}\right] \\
m_{\emptyset}: & I & \longrightarrow & \star[\varnothing]
\end{array}
$$

This basic observation has the remarkable consequence that for every set $\mathscr{L}$ of labels:

Proposition 2: The asynchronous graph $\star[\mathscr{L}]$ comes equipped with the structure of a commutative monoid in the symmetric monoidal category (Asynch,,$I)$ ).

The multiplication and unit of the commutative monoid

$$
\begin{array}{cccc}
\text { mult }_{\mathscr{L}}: & \star[\mathscr{L}] \amalg \star[\mathscr{L}] & \longrightarrow & \star[\mathscr{L}] \\
\text { unit }_{\mathscr{L}}: & I & \longrightarrow & \star[\mathscr{L}]
\end{array}
$$

are defined by transporting along the symmetric monoidal functor $t[-]$ the commutative monoid structure $\left(\mathscr{L}, \nabla_{\mathscr{L}}, \mathbf{0}_{\mathscr{L}}\right)$ 
of the original set $\mathscr{L}$ of labels, provided by the canonical codiagonal and zero functions

$$
\begin{aligned}
& \nabla_{\mathscr{L}}: \mathscr{L}+\mathscr{L} \longrightarrow \mathscr{L} \\
& \mathbf{0}_{\mathscr{L}}: \quad \varnothing \quad \longrightarrow \mathscr{L}
\end{aligned}
$$

in the cocartesian category $($ Set,,$+ \varnothing)$.

Asynchronous games: Following the track and spirit of [23], we define an asynchronous game $\left(G, \diamond_{G}, \lambda_{G}\right)$ as an asynchronous graph $\left(G, \diamond_{G}\right)$ where every edge $m: x \rightarrow y$ is seen as a move between positions $x, y$ "polarized" by a label $\boldsymbol{P}$ when the move $m$ is played by Player on the side of the proof (or the program) and by a label $\boldsymbol{O}$ when the move $m$ is played by Opponent on the side of the refutation (or the environment). The polarization of moves is moreover required to be consistent with permutations. For that reason, an asynchronous game $\left(G, \diamond_{G}, \lambda_{G}\right)$ is the same thing as an asynchronous graph $\left(G, \diamond_{G}\right)$ equipped with an asynchronous graph homomorphism

$$
\lambda_{G} \quad: \quad\left(G, \diamond_{G}\right) \longrightarrow \star[\boldsymbol{O}, \boldsymbol{P}] .
$$

In this formulation, the tensor product of two asynchronous games $\left(G, \diamond_{G}, \lambda_{G}\right)$ and $\left(H, \diamond_{H}, \lambda_{H}\right)$ is defined as the asynchronous graph $\left(G, \diamond_{G}\right) ш\left(H, \diamond_{H}\right)$ with labelling map $\lambda_{G \uplus H}$ defined as the composite of the tensor product of the two labelling functions

$$
\left(G, \diamond_{G}\right) \amalg\left(H, \diamond_{H}\right) \stackrel{\lambda_{G} \uplus \lambda_{H}}{\longrightarrow} \star[\boldsymbol{O}, \boldsymbol{P}] ш \star[\boldsymbol{O}, \boldsymbol{P}]
$$

followed by the asynchronous graph homomorphism

$$
\star[\boldsymbol{O}, \boldsymbol{P}] \amalg \star[\boldsymbol{O}, \boldsymbol{P}] \stackrel{\text { mult }}{\longrightarrow} \star[\boldsymbol{O}, \boldsymbol{P}]
$$

defined by the multiplication of the asynchronous graph $\star[\boldsymbol{O}, \boldsymbol{P}]$ described in $(11)$ which associates to each polarity $\boldsymbol{O}$ or $\boldsymbol{P}$ of the domain the same polarity $\boldsymbol{O}$ or $\boldsymbol{P}$ of the codomain. This definition of the tensor product $A, B \mapsto A \otimes B$ of two asynchronous games is reminiscent of a very similar construction in the template game model of differential linear logic [23], [24] which we briefly recall now.

Template games: The basic idea of template game semantics is to define a game $\left(A, \lambda_{A}\right)$ as a pair consisting $(a)$ of a category $A$ whose objects are the positions $x, y \in A$ of the game, and whose morphisms $f: x \rightarrow y$ are the trajectories or plays of the game, and $(b)$ of a labelling functor

$$
\lambda_{A}: A \longrightarrow t_{\text {game }}
$$

to a specific category $t_{\text {game }}$ of polarities called the template of games. One main advantage of the definition is that the template $t_{\text {game }}$ may be chosen to describe a particular class of games (sequential, concurrent) and scheduling policy between Player and Opponent (alternating, non-alternating). Typically, in the case of non-alternating concurrent games, the template of games $t_{\text {game }}=t_{\text {conc }}$ is defined as the category with one object $\langle *\rangle$ generated by two maps

$$
P \subset\langle *\rangle \longmapsto o
$$

and the unique equation $\boldsymbol{P} \cdot \boldsymbol{O}=\boldsymbol{O} \cdot \boldsymbol{P}$. It is worth observing that since a category with one object is the same thing as a monoid, the category $t_{\text {game }}=t_{\text {conc }}$ may be equivalently defined as the commutative monoid freely generated by the two elements $\{\boldsymbol{O}, \boldsymbol{P}\}$. More generally, it makes sense to consider the symmetric monoidal functor

$$
\star\{-\mid\} \quad: \quad(\text { Set },+, \varnothing) \longrightarrow(\text { Cat }, \times, \mathbf{1})
$$

which transports every set $\mathscr{L}$ to the commutative monoid $t\{\mathscr{L}\}$ freely generated by $\mathscr{L}$, seen as a category with one object. As a consequence, every category $t\{\mathscr{L}\}$ defines a commutative monoid in Cat with multiplication and unit noted

$$
\begin{aligned}
& \text { mult }_{\mathscr{L}}: \star \star\{\mathscr{L}\} \times \star\{\mathscr{L}\} \rightarrow \star\{\{\mathscr{L}\} \\
& \text { unit } \mathscr{L}: \quad 1 \quad t \quad t\{\mathscr{L}\}
\end{aligned}
$$

There is an obvious parallel with asynchronous games here, since every concurrent template game $A$ is a category labelled by the category of polarities

$$
\star_{\text {game }}=\star\{|\boldsymbol{O}, \boldsymbol{P}|\}
$$

in the same way that every asynchronous game $\left(G, \diamond_{G}, \lambda_{G}\right)$ is an asynchronous graph $\left(G, \diamond_{G}\right)$ labelled by the asynchronous graph of polarities $\star[\boldsymbol{O}, \boldsymbol{P}]$.

Template games continued: One key observation of [23] is that in good situations, the template of games $t_{\text {game }}$ comes equipped with a companion category $t_{\text {strat }}$ called the template of strategies, whose purpose is to describe the possible schedulings of a strategy $\sigma$ between two template games $\left(A, \lambda_{A}\right)$ and $\left(B, \lambda_{B}\right)$. The category $t_{\text {strat }}$ comes moreover equipped with two functors

$$
t_{\text {game }} \longleftarrow s
$$

which describe how the scheduling of the strategy $\sigma$ is related to the schedulings of the games $\left(A, \lambda_{A}\right)$ and $\left(B, \lambda_{B}\right)$. A strategy $\sigma$ between two games $\left(A, \lambda_{A}\right)$ and $\left(B, \lambda_{B}\right)$

$$
\sigma=\left(S, s, t, \lambda_{\sigma}\right):\left(A, \lambda_{A}\right) \longrightarrow\left(B, \lambda_{B}\right)
$$

is defined as a span of functors

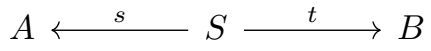

with support a category $S$, together with a functor $\lambda_{\sigma}: S \rightarrow$ $t_{\text {strat }}$ making the diagram below commute:

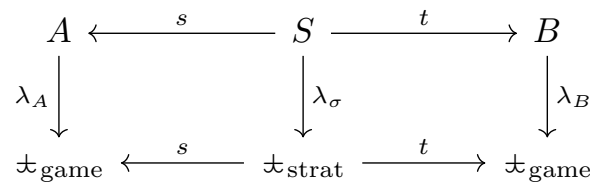

In the case of concurrent template games, where $t_{\text {game }}=$ $\star\{\boldsymbol{O}, \boldsymbol{P}\}$, the template of strategies is defined as the category

$$
t_{\text {strat }}=t\left\{\boldsymbol{O}_{s}, \boldsymbol{P}_{s}, \boldsymbol{O}_{t}, \boldsymbol{P}_{t}\right\}
$$

The intuition is that every move $m$ played by a strategy $\sigma$ between two concurrent games $A$ and $B$ may be labelled in 
four different ways depending on the polarity of $m$ and the component $A$ or $B$ in which it is played:

$\boldsymbol{O}_{s}$ : an Opponent move played by $\sigma$ in the source $A$,

$\boldsymbol{P}_{s}:$ a Player move played by $\sigma$ in the source $A$,

$\boldsymbol{O}_{t} \quad$ : an Opponent move played by $\sigma$ in the $\operatorname{target} B$,

$\boldsymbol{P}_{t} \quad$ : a Player move played by $\sigma$ in the target $B$.

The functors $s$ and $t$ are then characterized by the image of the four generators of $t_{\text {strat }}=\star\left\{\mid \boldsymbol{O}_{s}, \boldsymbol{P}_{s}, \boldsymbol{O}_{t}, \boldsymbol{P}_{t}\right\}$, as follows:

$$
\begin{array}{lllll}
s: & \boldsymbol{O}_{s} \mapsto \boldsymbol{P} & \boldsymbol{P}_{s} \mapsto \boldsymbol{O} & \boldsymbol{O}_{t} \mapsto \mathrm{id}_{\langle *\rangle} & \boldsymbol{P}_{t} \mapsto \mathrm{id}_{\langle *\rangle} \\
t: \boldsymbol{O}_{s} \mapsto \mathrm{id}_{\langle *\rangle} & \boldsymbol{P}_{s} \mapsto \mathrm{id}_{\langle *\rangle} & \boldsymbol{O}_{t} \mapsto \boldsymbol{O} & \boldsymbol{P}_{t} \mapsto \boldsymbol{P}
\end{array}
$$

Note that the definition of $s$ and $t$ formalizes the intuition that every move $m$ of polarity $\boldsymbol{O}_{s}$ or $\boldsymbol{P}_{s}$ played by the strategy $\sigma$ should have a reverse polarity $\boldsymbol{P}$ or $\boldsymbol{O}$ in the source game $A$, while a move $m$ of polarity $\boldsymbol{O}_{t}$ or $\boldsymbol{P}_{t}$ should retain its polarity $\boldsymbol{O}$ or $\boldsymbol{P}$ in the target game $B$.

A tale of deadlocks and diagonals: As we saw, there is a strong affinity between asynchronous games and concurrent template games. This is particularly striking in the definition of the tensor product of two concurrent template games $\left(A, \lambda_{A}\right)$ and $\left(B, \lambda_{B}\right)$ as the template game

$$
\left(A, \lambda_{A}\right) \otimes\left(B, \lambda_{B}\right)=\left(A \times B, \lambda_{A \otimes B}\right)
$$

with support the cartesian product $A \times B$ of the categories $A$ and $B$, and with labelling functor $\lambda_{A \otimes B}$ defined as the composite of the cartesian product of the two labelling functors

$$
A \times B \stackrel{\lambda_{A} \times \lambda_{B}}{\longrightarrow} \star\{\{\boldsymbol{O}, \boldsymbol{P}\} \times \star\{\boldsymbol{O}, \boldsymbol{P}\}
$$

followed by the multiplication functor defined in 16

$$
\star\{|\boldsymbol{O}, \boldsymbol{P}|\} \times \star\{\boldsymbol{O}, \boldsymbol{P}\} \stackrel{\text { mult }_{\mathscr{L}}}{\longrightarrow} \star\{\{\boldsymbol{O}, \boldsymbol{P}\}
$$

for the category $t\{\{\mathscr{L}\}$ where $\mathscr{L}=\{\boldsymbol{O}, \boldsymbol{P}\}$. However, one main difference between (24) and (12) is that the cartesian product of categories replaces the shuffle tensor product of asynchronous graphs. Let us illustrate the difference with an example, and explain why the interpretation provided by the asynchronous game model is more satisfactory than the concurrent template model. Consider the asynchronous game $A$ consisting of one Opponent move $m: x \rightarrow x^{\prime}$ between two positions $x, x^{\prime}$; and the asynchronous game $B$ consisting of one Player move $n: y \rightarrow y^{\prime}$ between two positions $y, y^{\prime}$. The asynchronous game $A \otimes B$ has the asynchronous graph with four vertices (positions), four edges (moves) and a permutation tile as support:

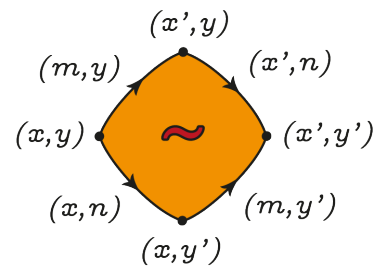

Now, consider the strategy $\sigma$ of the game $A \amalg B$ which plays the trajectory (and its prefixes)

$$
(x, y) \stackrel{(m, y)}{\longrightarrow}\left(x^{\prime}, y\right) \stackrel{\left(x^{\prime}, n\right)}{\longrightarrow}\left(x^{\prime}, y^{\prime}\right)
$$

from the starting position $(x, y)$ and the counter-strategy $\tau$ which plays the trajectory (and its prefixes)

$$
(x, y) \stackrel{(x, n)}{\longrightarrow}\left(x, y^{\prime}\right) \stackrel{\left(m, y^{\prime}\right)}{\longrightarrow}\left(x^{\prime}, y^{\prime}\right)
$$

from the very same position $(x, y)$. In this situation, the interaction between $\sigma$ and $\tau$ produces a deadlock, because each strategy $\sigma$ and $\tau$ is waiting for a signal which never comes from the other side in order to proceed. The deadlock between $\sigma$ and $\tau$ is nicely reflected in the asynchronous game $A \amalg B$ by the fact that the intersection of $\sigma$ and of $\tau$ only contains the empty path from $(x, y)$ to itself.

Unfortunately, the situation is not as satisfactory when one shifts to the corresponding concurrent template games $A$ and $B$. Indeed, when one considers $\sigma$ and $\tau$ as strategies playing on the concurrent template game $A \otimes B$ obtained by tensoring $A$ and $B$ using $(23)$, it appears that the two trajectories 26) and (27) are identified as the "synchronized move" given by the diagonal map $(m, n):(x, y) \rightarrow\left(x^{\prime}, y^{\prime}\right)$ of the cartesian category $A \times B$ underlying the concurrent template game $A \otimes B$, which we represent in full below:

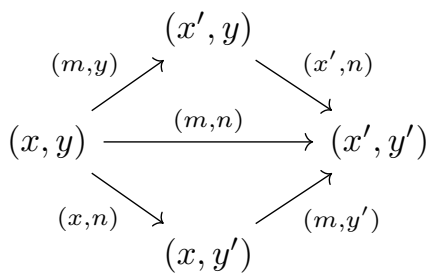

The fact that the two trajectories (26) and 27) are identified to the diagonal map $(m, n)$ means that the intersection of $\sigma$ and $\tau$ is not trivial anymore, since it contains the diagonal map. In a sense, the categorical interpretation believes (wrongly!) that the two strategies $\sigma$ and $\tau$ could "resolve" the deadlock by playing the two moves $m$ and $n$ synchronously. This excess of synchronization in the interpretation of $A \otimes B$ should be seen as a defect (not as a feature!) of the original concurrent template game semantics formulated in [23], [24].

The Gray tensor product: We correct the situation in the present paper by shifting one dimension higher the original formalism of template game semantics: we design an asynchronous template game model where template games $\left(A, \lambda_{A}\right)$ are defined as 2-categories instead of simply as 1-categories. To that purpose, we take seriously the pretty striking analogy between the shuffle tensor product $A, B \mapsto A \amalg B$ of asynchronous graphs, and the Gray tensor product $A, B \mapsto A \bigotimes B$ of 2-categories. The Gray tensor product was introduced by Gray in [15] and it plays a fundamental role in contemporary categorical algebra, see for instance [4], [6]. One reason is that the Gray tensor product comes with a closed structure provided by the hom-2-category $[A, B]_{p s}$ between 2-categories, defined as the 2-category of 2-functors from $A$ to $B$, pseudonatural transformations and modifications. Another reason is a subtle coherence theorem for tricategories [13], [16] establishes that every tricategory is equivalent to a Gray-category, defined as category enriched over the category 2-Cat equipped with the Gray tensor product. 
One first contribution of the paper is to clarify the relationship between the shuffle tensor product of asynchronous graphs and the Gray tensor product of 2-categories by constructing a symmetric monoidal functor

$$
\langle-\rangle:(\text { Asynch }, \amalg, \mathbf{I}) \longrightarrow(\mathbf{2} \text {-Cat }, \bigotimes, \mathbf{I})
$$

The functor $\langle-\rangle$ associates to every asynchronous graph $(G, \diamond)$ the 2-category $\langle G, \diamond\rangle$ whose objects are the vertices of $G$, whose morphisms are the paths of $G$ and whose 2-dimensional cells are the reshufflings (or reschedulings) between paths of the graph $G$. A detailed definition of reshuffling appears in $\$ \mathrm{~V}-\mathrm{A}$ but let us already mention that the main purpose of reshufflings is to identify different combinations of permutation tiles such as

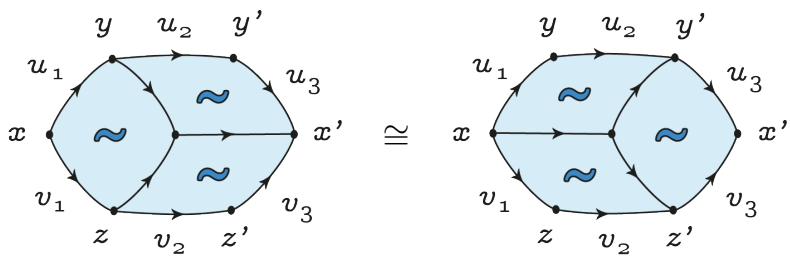

which deserve to be considered as equivalent in the asynchronous graph $G$. The fact that the functor $\langle-\rangle$ comes equipped with natural isomorphisms of 2-categories:

$$
\begin{array}{cccc}
p_{G, H}: & \left\langle G, \diamond_{G}\right\rangle \otimes\left\langle H, \diamond_{H}\right\rangle & \longrightarrow & \left\langle G \amalg H, \diamond_{G \amalg H}\right\rangle \\
p_{I}: & \mathbf{1} & \longrightarrow & \langle\mathbf{I}\rangle
\end{array}
$$

qualifies the Gray tensor product as a natural and expressive extension of the usual shuffle product of asynchronous graphs. In particular, the functor $\langle-\rangle$ may be composed to $t[-]$ in order to obtain the symmetric monoidal functor

$$
\star\{-\}:(\text { Set },+, \varnothing) \longrightarrow(\text { 2-Cat }, \times, 1)
$$

which associates to every set $\mathscr{L}$ the symmetric monoidal category freely generated by $\mathscr{L}$, seen as a 2-category with one object. Note that $t\{|-|\}$ plays the same role as the functor 15 which it upgrades from categories to 2-categories.

Asynchronous template games: We have accumulated enough material in order to define the notion of asynchronous template game. There is still one fundamental obstruction which we need to resolve however: the notion of template formulated in [23], [24] is defined as an internal category

$$
\star=(\star[0], \star[1], \text { mult }: \star[2] \rightarrow \star[1], \text { unit }: \star[1] \rightarrow \star[0])
$$

in the category $\mathbb{S}$ with finite limits, where $t[0]=t_{\text {game }}$ is the object of objects and $t[1]=t_{\text {strat }}$ is the object of morphisms. For that reason, the formalism of template games originally developed in [23], [24] does not work any more when the category $\mathbb{S}=$ Cat with finite limits is replaced by the monoidal category $\mathbb{S}=\mathbf{2}$-Cat equipped with the Gray tensor product.

One main contribution of the paper is to resolve that foundational issue by establishing an unexpected connection with the seminal work by Marcelo Aguiar on quantum groups [3].
The key observation is that the 2-category $t\{\boldsymbol{O}, \boldsymbol{P}\}$ of asynchronous polarities is equipped with a comonoid structure

$$
\begin{gathered}
d: \star\{|\boldsymbol{O}, \boldsymbol{P}|\} \\
e: t\{\{\boldsymbol{O}, \boldsymbol{P} \mid\} \longrightarrow \mathbf{1}
\end{gathered}
$$

where we find useful to recall the isomorphism:

$$
\star\left\{\mid \boldsymbol{O}_{1}, \boldsymbol{P}_{1}\right\} \otimes \star\left\{\boldsymbol{O}_{2}, \boldsymbol{P}_{2}\right\} \cong \quad ‡\left\{\boldsymbol{O}_{1}, \boldsymbol{P}_{1}, \boldsymbol{O}_{2}, \boldsymbol{P}_{2}\right\}
$$

associated to the symmetric monoidal functor (30). The comultiplication $d$ is then characterized (and defined) by the fact that $d$ transports the edges $\boldsymbol{O}$ and $\boldsymbol{P}$ to the paths of length 2:

$$
d \quad: \quad \boldsymbol{O} \mapsto \boldsymbol{O}_{2} \cdot \boldsymbol{O}_{1} \quad \boldsymbol{P} \mapsto \boldsymbol{P}_{1} \cdot \boldsymbol{P}_{2}
$$

Note the right-to-left orientation of the Opponent polarity $\boldsymbol{O}$ and the left-to-right orientation of the Player polarity $\boldsymbol{P}$. A nice connection emerges here between the notion of copycat strategy in game semantics and the notion of Gray comonoid defined as a comonoid for the Gray tensor product $\triangle$ of 2categories: typically, the path or trajectory

$$
\boldsymbol{O} \cdot \boldsymbol{P} \cdot \boldsymbol{O} \in \quad \star\{\{\boldsymbol{O}, \boldsymbol{P}\}
$$

describing the sequence of an Opponent move, a Player move and an Opponent move played in the 2-category $\star\{|\boldsymbol{O}, \boldsymbol{P}|\}$ of polarities, is transported to the path or trajectory

$$
\boldsymbol{O}_{2} \cdot \boldsymbol{O}_{1} \cdot \boldsymbol{P}_{1} \cdot \boldsymbol{P}_{2} \cdot \boldsymbol{O}_{2} \cdot \boldsymbol{O}_{1} \quad \in \quad t\left\{\mid \boldsymbol{O}_{1}, \boldsymbol{P}_{1}\right\} \otimes \star\left\{\mid \boldsymbol{O}_{2}, \boldsymbol{P}_{2}\right\}
$$

of six moves shuffled between $t\left\{\boldsymbol{O}_{1}, \boldsymbol{P}_{1}\right\}$ and $t\left\{\boldsymbol{O}_{2}, \boldsymbol{P}_{2}\right\}$. The six moves may be represented as blue nodes and red nodes on a string diagram, where the "zig-zag" induced by the comultiplication $d$ implements the scheduling of the usual copycat strategy [1], [19] of game semantics:

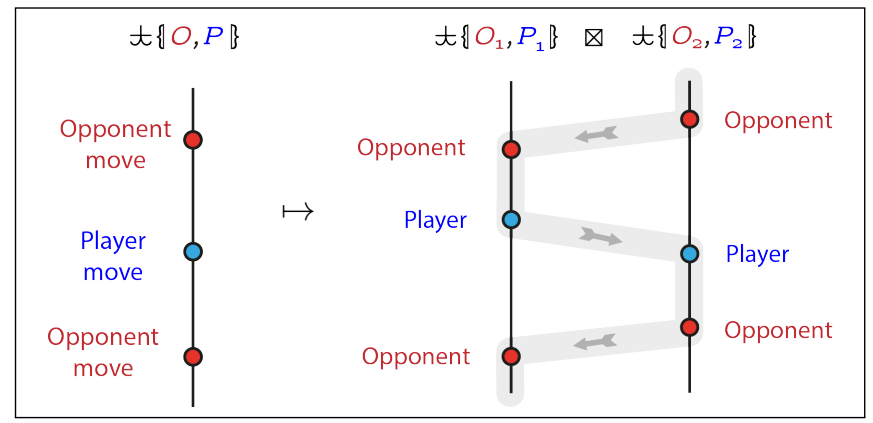

Note that following a well-established convention of game semantics, the flow of time goes top-down, as indicated by the grey arrows switching components in the diagram. This connection between Gray comonoids and game semantics can be explained by the existence of a canonical 2-functor

$$
q_{A, B} \quad: \quad A \otimes B \longrightarrow A \times B
$$

and the fact that the composite

$$
A \stackrel{d_{A}}{\longrightarrow} A \otimes A \stackrel{q_{A, B}}{\longrightarrow} A \times A
$$

coincides for every Gray comonoid $\left(A, d_{A}, e_{A}\right)$ with the diagonal of the 2-category $A$. For this reason, the comultiplication $d_{A}$ of a Gray comonoid $A$ is required to provide a specific recipe to transport every morphism $f: x \rightarrow y$ in $A$ to 
a morphism $d_{A}(f):(x, x) \rightarrow(y, y)$ in $A \otimes A$ which projects as $f: x \rightarrow y$ on both left and right component. For that reason, one establishes easily that

Proposition 3: Given a set $S$, a Gray comonoid structure $\left(\star\{S\}, d_{S}, e_{S}\right)$ on the 2-category $\star\{S\}$ is the same thing as a polarity function $\lambda_{S}: S \rightarrow\{\boldsymbol{O}, \boldsymbol{P}\}$.

The polarity $\lambda_{S}(e)$ for $e \in S$ indicates whether the morphism $d(e):(*, *) \rightarrow(*, *)$ in the 2-category $t\{S\}\} \otimes \star\{\mid S\}$ is defined left-to-right as $e_{1} \cdot e_{2}$ in the case $\lambda_{S}(e)=\boldsymbol{P}$ or rightto-left as $e_{2} \cdot e_{1}$ in the case $\lambda_{S}(e)=\boldsymbol{O}$.

This pleasant convergence between the notions of polarity in a game and of Gray comonoid leads us to the main definition of the paper:

Definition 1 (Asynchronous template game): An asynchronous template game $\left(A, \lambda_{A}\right)$ is defined as a Gray comonoid $\left(A, d_{A}, e_{A}\right)$ equipped with a Gray comonoid homomorphism

$$
\lambda_{A}: A \longrightarrow \star\{\boldsymbol{O}, \boldsymbol{P}\} .
$$

to the Gray comonoid $t\{\{\boldsymbol{O}, \boldsymbol{P}\}$ in 31 . The 2-category $A$ is called the support of the asynchronous template game $\left(A, \lambda_{A}\right)$. Here, by Gray comonoid homomorphism

$$
h:\left(A, d_{A}, e_{A}\right) \longrightarrow\left(B, d_{B}, e_{B}\right)
$$

we mean a 2-functor $h: A \rightarrow B$ between the underlying 2-categories $A$ and $B$, making the diagram below commute:

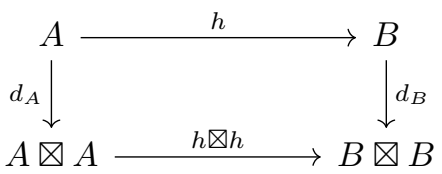

The definition (Def. 1) of asynchronous template game provides a conservative extension of the traditional notion of asynchronous games, in the sense that an asynchronous game in the usual sense (described earlier in the paper) happens to be the same thing as an asynchronous template game $\left(A, \lambda_{A}\right)$ whose support $A$ is the 2-category $A=\left\langle G, \diamond_{G}\right\rangle$ of positions, trajectories and reshufflings of an asynchronous graph $\left(G, \diamond_{G}\right)$.

The template of asynchronous games: At this stage, we want to upgrade one dimension up the definitions (17) and (21) of the template of concurrent games in [23]. We thus write

$$
\star_{\text {game }}=\star\{\boldsymbol{O}, \boldsymbol{P}\} \quad \star_{\text {strat }}=\star\left\{\left\{\boldsymbol{O}_{s}, \boldsymbol{P}_{s}, \boldsymbol{O}_{t}, \boldsymbol{P}_{t}\right\}\right.
$$

where $t_{\text {game }}$ and $t_{\text {strat }}$ are now understood as 2-categories of polarities, instead of categories. We have seen in Def. 1 that a template game $\left(A, \lambda_{A}\right)$ is a Gray comonoid $\left(A, d_{A}, e_{A}\right)$ equipped with a comonoid homomorphism

$$
\lambda_{A}: A \longrightarrow t_{\text {game }}
$$

There remains to define the appropriate asynchronous notion of strategy $\sigma$ adapting the original definition (19) as a span of categories. Following the guidance and inspiration of [3], we observe that $t_{\text {strat }}$ comes equipped with a 2 -functor

$$
\text { coact }_{t}: t_{\text {strat }} \longrightarrow t_{\text {game }} \otimes t_{\text {strat }} \otimes t_{\text {game }}
$$

defined by the images of the four generating edges:

$$
\boldsymbol{O}_{s} \mapsto \boldsymbol{P}_{1} \cdot \boldsymbol{O}_{s} \quad \boldsymbol{P}_{s} \mapsto \boldsymbol{P}_{s} \cdot \boldsymbol{O}_{1} \quad \boldsymbol{O}_{t} \mapsto \boldsymbol{O}_{3} \cdot \boldsymbol{O}_{t} \quad \boldsymbol{P}_{t} \mapsto \boldsymbol{P}_{t} \cdot \boldsymbol{P}_{3}
$$

where the indices 1 and 3 indicate in which component the moves are played. Here, the 2-functor coact $_{\star}$ should be understood as an asynchronous variant (and refinement) of the span $(s, t)$ of projection functors $(18)$ as defined in 22. Moreover, one observes that the 2-functor coact ${ }_{\star}$ just defined satisfies the equations required of a $t_{\text {game }}, t_{\text {game}}$-bicomodule structure on the template 2-category $t_{\text {strat }}$ of strategies, see $\$ \overline{V I-B}$ for a definition of bicomodule between comonoids.

This observation leads us to the following definition of asynchronous strategy between template games:

Definition 2 (Asynchronous strategies): An asynchronous strategy

$$
\sigma=\left(S, \operatorname{coact}_{\sigma}, \lambda_{\sigma}\right):\left(A, \lambda_{A}\right) \longrightarrow\left(B, \lambda_{B}\right)
$$

is a triple $\left(S\right.$, coact $\left._{\sigma}, \lambda_{\sigma}\right)$ consisting of 2-category $S$ used as support, together with an $A, B$-bicomodule structure

$$
\operatorname{coact}_{\sigma} \quad: \quad S \longrightarrow A \otimes S \otimes B
$$

and a polarity 2 -functor

$$
\lambda_{\sigma} \quad: \quad S \longrightarrow \star_{\text {strat }}
$$

making the diagram below commute:

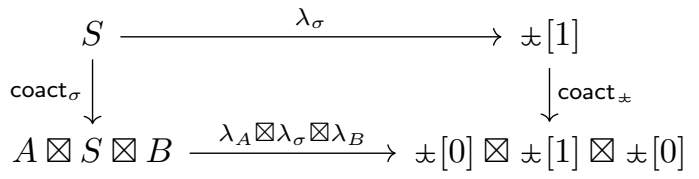

As explained later in the paper, writing

$$
\star_{\text {asynch }}=\left(\star_{\text {game }}, \star_{\text {strat }}, \text { coact }_{t}\right)
$$

for the template of asynchronous games just described, we obtain a symmetric monoidal closed (and in fact $*$-autonomous) bicategory Games $\left(t_{\text {asynch }}\right)$ of asynchronous template games and asynchronous strategies between them.

Theorem 1: The bicategory Games( $\left.\star_{\text {asynch }}\right)$ is *autonomous and cartesian, and thus defines a model of the multiplicative and additive fragment of linear logic (MALL).

On the practical side, the tensor product $A, B \mapsto A \otimes B$ of asynchronous template games is defined in $\operatorname{Games}\left(\star_{\text {asynch }}\right)$ using the Gray tensor product and thus avoids the defect discussed in (25) and (28). On the conceptual side, the construction extends in a very natural way the original framework of template games [23], [24] to the more general situation where the template $t=t_{\text {asynch }}$ defines an internal category in a monoidal category $\mathbb{S}$ such as $\mathbb{S}=(\mathbf{2}$-Cat, $\mathbb{\nabla}, \mathbf{1})$ - see Aguiar [3] and $\$ \sqrt{\mathrm{VI}}, \$ \sqrt{\mathrm{VII}}$ for a definition - instead of an internal category in a category $\mathbb{S}$ with limits such as $\mathbb{S}=$ Cat. 


\section{RELATED WORKS AND SYNOPSIS}

Besides the numerous connections already mentioned to the work on template games by Melliès [23], [24], we should mention the formal analysis of template games by Eberhard, Hirschowitz and Laouar [11] where a template $t$ is identified in full generality as a formal monad

$$
\star=(\star[0], \star[1], \text { mult, unit })
$$

living in a weak double category. Our construction follows that track by defining in $\$ \mathrm{VII}$ a template as a monad $t$ living in the weak double category of bicomodules $\operatorname{Comod}(\mathbb{S})$ in a monoidal category $(\mathbb{S}, \mathbb{\bigotimes}, \mathbf{1})$ with coreflexive equalizers preserved by the tensor product $\otimes$ componentwise. This is precisely the definition of internal category formulated by Aguiar in his seminal work on quantum algebras [3].

The present work is part of a broader trend of research on game semantics and concurrency, started with the definition of concurrent games by Abramsky and Melliès [2] and the subsequent series of works on asynchronous games [22], [21], [25] defined as asynchronous graphs (or event structures) with transitions polarized in $\{\boldsymbol{O}, \boldsymbol{P}\}$. The insight was adapted by Rideau and Winskel [28], [7] to the language of event structures, giving rise to very interesting developments by Castellan, Clairambault and Winskel [8], [9] on concurrent games based on event structures with symmetries. The notion of asynchronous template game based on 2-categories (see Def. 1) offers an expressive and powerful generalization of traditional asynchronous games based on event structures, and it would be interesting to understand whether the concurrent game model with symmetries formulated in [8], [9] can be accommodated in the language of asynchronous template games developed in the present paper.

Finally, we would like to mention the work by Eberhart and Hirschowitz [10] on a general theory of game semantics based on polynomial functors between presheaf or sheaf categories. We would be interested to see how their structural approach to game semantics could be adapted to the 2-categorical and monoidal framework of asynchronous template games developed in the present paper.

Synopsis of the paper: After a long and detailed overview of the paper which we found clarifying and mandatory in $\$ \Phi$ and a comparison with related works in $\$ \Pi$, we start the technical part of the paper with a precise and formal definition of asynchronous graphs in \$III We then recall the notion of Gray tensor product in \$IV and establish the important property that $\nabla$ preserves the coreflexive equalizers of 2-Cat componentwise. We then give an explicit description in $\$ \mathrm{~V}$ of the translation $(G, \diamond) \mapsto\langle G, \diamond\rangle$ from asynchronous graphs to 2-categories. Using the preservation of coreflexive equalizers established in $\$[\mathrm{IV}]$ we construct in $\$ \mathrm{VI}$ the weak double category of bicomodules $\operatorname{Comod}(\mathbb{S})$ in the symmetric monoidal category $\mathbb{S}=(\mathbf{2}$-Cat $, \mathbb{Q}, \mathbf{1})$. We finally define in $\$ \mathrm{VII}$ the template $t=t_{\text {asynch }}$ of asynchronous games, and establish in $\$$ VIII that the resulting bicategory Games $\left(\star_{\text {asynch }}\right)$ is symmetric monoidal closed, and in fact $*$-autonomous. As a cartesian and co-cartesian $*$-autonomous bicategory, it defines a 2-categorical model of the multiplicative additive fragment (MALL) of linear logic, where formula are interpreted as asynchronous template games and proofs as strategies between them. We conclude and indicate future directions in $\$ \mathrm{IX}$.

\section{ASYNCHRONOUS GRAPHS}

We start by describing in \$II-A the specific notion of asynchronous graph which we shall use in the paper. This leads us to define in $\$$ III-B the category Asynch of asynchronous graphs and of morphisms between them. Every asynchronous graph $(G, \diamond)$ may be seen as a presentation by generators and relations of a specific 2-category noted $\langle G, \diamond\rangle$. We construct a symmetric monoidal category of asynchronous graphs.

\section{A. Asynchronous graphs}

A square in a graph $G$ is defined as a pair $(p, q)$ of paths $p, q: x \rightarrow y$ of length 2 , with the same source $x$ and the same target $y$. An asynchronous graph $(G, \diamond)$ is a graph $G$ equipped with a set $\diamond$ of squares, satisfying a number of additional properties described below. We use the notation $p \diamond q$ when the square $(p, q)$ is an element of $\diamond$ and say in that case that the square $(p, q)$ defines a permutation tile. A permutation tile $u_{1} \cdot u_{2} \diamond v_{1} \cdot v_{2}$ between the paths $p=u_{1} \cdot u_{2}$ and $q=v_{1} \cdot v_{2}$ is depicted in the following way, as a 2-dimensional surface or tile between the paths $p$ and $q$ :

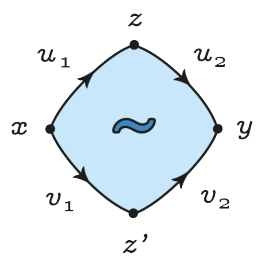

An asynchronous graph $(G, \diamond)$ is required to satisfy the following properties:

1. every permutation tile is symmetric: for all paths $p, q$ : $x \rightarrow y$ of length 2 with same source $x$ and same target $y, p \diamond q$ implies that $q \diamond p$,

2. every permutation tile is deterministic: for all paths $p, q, q^{\prime}: x \rightarrow y$ of length 2 with same source $x$ and same target $y, p \diamond q$ and $p \diamond q^{\prime}$ implies that $q=q^{\prime}$,

3. the cube property: for all pairs $p, q: x \rightarrow y$ of paths of length 3 with same source $x$ and same target $y$, noted $p=$ $u_{1} \cdot u_{2} \cdot u_{3}$ and $q=v_{1} \cdot v_{2} \cdot v_{3}$, there are edges $w_{3}, u_{2}^{\prime}, v_{2}^{\prime}$ and a sequence of permutation tiles

$$
u_{2} \cdot u_{3} \diamond u_{2}^{\prime} \cdot w_{3} \quad u_{1} \cdot u_{2}^{\prime} \diamond v_{1} \cdot v_{2}^{\prime} \quad v_{2}^{\prime} \cdot w_{3} \diamond v_{2} \cdot v_{3}
$$

if and only if there are edges $w_{1}, u_{2}^{\prime \prime}, v_{2}^{\prime \prime}$ and a sequence of permutation tiles

$$
u_{1} \cdot u_{2} \diamond w_{1} \cdot u_{2}^{\prime \prime} \quad u_{2}^{\prime \prime} \cdot u_{3} \diamond v_{2}^{\prime \prime} \cdot v_{3} \quad w_{1} \cdot v_{2}^{\prime \prime} \diamond v_{1} \cdot v_{2} .
$$

The cube property is nicely described by the following picture:

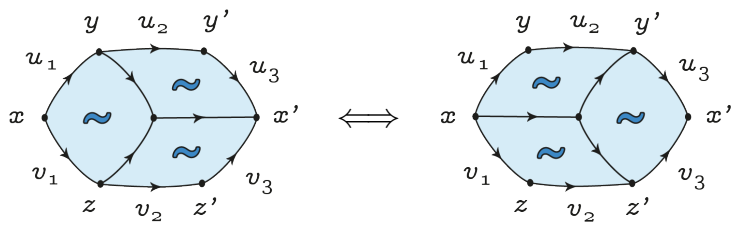




\section{B. The category of asynchronous graphs}

In this section, we define the category Asynch of asynchronous graphs and establish that the category has all finite limits. An asynchronous homomorphism between asynchronous graphs is defined as

Definition 3: An asynchronous graph homomorphism, or asynchronous homomorphism,

$$
f \quad: \quad\left(G, \diamond_{G}\right) \longrightarrow\left(H, \diamond_{H}\right)
$$

is a graph homomorphism $f: G \rightarrow H$ between the underlying graphs, such that

$$
u_{1} \cdot u_{2} \diamond_{G} v_{1} \cdot v_{2} \Rightarrow f\left(u_{1}\right) \cdot f\left(u_{2}\right) \diamond_{H} f\left(v_{1}\right) \cdot f\left(v_{2}\right)
$$

for every pair of paths $p=u_{1} \cdot u_{2}$ and $q=v_{1} \cdot v_{2}$ of length 2 with same source and target.

The category Asynch is defined in the following way: its objects are the asynchronous graphs and its morphisms are the asynchronous homomorphisms between them.

\section{Finite limits of asynchronous graphs}

We establish now that the category Asynch has all finite limits. We proceed in two steps. A preliminary observation is that the category Asynch is cartesian, with cartesian product defined as

$$
\left(G, \diamond_{G}\right) \times\left(H, \diamond_{H}\right)=\left(G \times H, \diamond_{G \times H}\right)
$$

where $G \times H$ denotes the cartesian product of the underlying graphs $G$ and $H$, and where the set of permutation tiles $\diamond_{G \times H}=\diamond_{G} \times \diamond_{H}$ is defined as expected: every pair of paths

$$
p, q:\left(x, x^{\prime}\right) \rightarrow\left(y, y^{\prime}\right)
$$

of length 2 in the graph $G \times H$ can be written as

$$
\begin{aligned}
& p=\left(x, x^{\prime}\right) \stackrel{\left(u_{1}, u_{1}^{\prime}\right)}{\longrightarrow}\left(z_{1}, z_{1}^{\prime}\right) \stackrel{\left(u_{2}, u_{2}^{\prime}\right)}{\longrightarrow}\left(y, y^{\prime}\right) \\
& q=\left(x, x^{\prime}\right) \stackrel{\left(v_{2}, v_{1}^{\prime}\right)}{\longrightarrow}\left(z_{2}, z_{2}^{\prime}\right) \stackrel{\left(y, y^{\prime}\right)}{\longrightarrow}
\end{aligned}
$$

One thus declares that the two paths $p$ and $q$ above define a permutation tile $p \diamond_{G \times H} q$ precisely when their projections define permutation tiles in $\left(G, \diamond_{G}\right)$ and in $\left(H, \diamond_{H}\right)$ :

$$
u_{1} \cdot u_{2} \diamond_{G} v_{1} \cdot v_{2} \quad \text { and } \quad u_{1}^{\prime} \cdot u_{2}^{\prime} \diamond_{H} v_{1}^{\prime} \cdot v_{2}^{\prime} .
$$

In other words,

$$
p \diamond_{G \times H} q \quad \Longleftrightarrow \quad \pi_{1}(p) \diamond_{G} \pi_{1}(q) \text { and } \pi_{2}(p) \diamond_{H} \pi_{2}(q)
$$

where $\pi_{1}: G \times H \rightarrow G$ and $\pi_{2}: G \times H \rightarrow H$ denote the two graph homomorphisms defined by projection. The terminal object of Asynch is the asynchronous graph $t[$ tic] with one single vertex $*$, one single edge tic : $* \rightarrow *$ and a permutation tile tic - tic $\diamond$ tic - tic which permutes the edge tic with itself.

We then establish in the Appendix $\$ \mathrm{~A}$ the less obvious property that the category Asynch has equalizers. From this follows that:

Proposition 4: The category Asynch has finite products and equalizers, and thus has all finite limits.

\section{THE GRAY TENSOR PRODUCT}

For the sake of completeness, we provide in the Appendix (see $\$ \mathrm{~B}$ ) a purely algebraic description by generators and relations of the Gray tensor product $\mathscr{A} \otimes \mathscr{B}$ of a pair of 2categories $\mathscr{A}$ and $\mathscr{B}$. The definition is somewhat involved however, and we thus find more convenient to describe below a characterization of the Gray tensor product $\mathscr{A} \otimes \mathscr{B}$ of two small 2-categories $\mathscr{A}, \mathscr{B}$ adapted from the work by Bourke and Gurski [6]. Using this specific formulation, we establish the main result of the section, which states that the Gray tensor product of 2-categories $\mathscr{A}, \mathscr{B} \mapsto \mathscr{A} \bigotimes \mathscr{B}$ preserves coreflexive equalizers componentwise.

\section{A. A concise characterization of the Gray tensor product}

The Gray tensor product $\mathscr{A}, \mathscr{B} \mapsto \mathscr{A} \otimes \mathscr{B}$ of 2-categories has the remarkable property that its unit coincides with the terminal 2-category 1 . From this follows that the Gray tensor product $\mathscr{A} \otimes \mathscr{B}$ of a pair of 2-categories $\mathscr{A}$ and $\mathscr{B}$ comes equipped with a pair of "projection" 2 -functors

$$
\mathscr{A} \cong \mathscr{A} \otimes \mathbf{1} \longleftarrow \pi_{1} \mathscr{A} \otimes \mathscr{B} \stackrel{\pi_{2}}{\longrightarrow} \mathbf{1} \otimes \mathscr{B} \cong \mathscr{B}
$$

which induce in turn a 2 -functor

$$
q_{\mathscr{A}, \mathscr{B}} \quad: \quad \mathscr{A} \otimes \mathscr{B} \longrightarrow \mathscr{A} \times \mathscr{B}
$$

The key observation at this stage is that the definition of Gray tensor product is carefully carved to ensure that this 2-functor is locally fully faithful. This means that for every pair of 1-cells

$$
(A, B) \underset{g}{\stackrel{f}{\rightleftharpoons}}\left(A^{\prime}, B^{\prime}\right)
$$

in the Gray tensor product $\mathscr{A} \otimes \mathscr{B}$, there is a one-to-one correspondence between the hom-sets

$$
\begin{gathered}
\mathscr{A} \otimes \mathscr{B}\left((A, B),\left(A^{\prime}, B^{\prime}\right)\right)(f, g) \\
\cong \mathscr{A}\left(A, A^{\prime}\right)\left(f_{1}, g_{1}\right) \times \mathscr{A}\left(B, B^{\prime}\right)\left(f_{2}, g_{2}\right)
\end{gathered}
$$

where the morphisms $f_{1}, g_{1}$ of $\mathscr{A}$ and $f_{2}, g_{2}$ of $\mathscr{B}$ are defined by projection:

$$
A \underset{g_{1}=\pi_{1}(g)}{\stackrel{f_{1}=\pi_{1}(f)}{\longrightarrow}} A^{\prime} \quad B \underset{g_{2}=\pi_{2}(g)}{\stackrel{f_{2}=\pi_{2}(f)}{\longrightarrow}} B^{\prime}
$$

In other words, a 2-cell in the 2-category $\mathscr{A} \otimes \mathscr{B}$ of the form

$$
\theta \quad: \quad f \Longrightarrow g \quad: \quad(A, B) \longrightarrow\left(A^{\prime}, B^{\prime}\right)
$$

may be equivalently defined as a pair

$$
\begin{aligned}
& \theta_{1}: f_{1} \longrightarrow g_{1}: A \longrightarrow A^{\prime} \\
& \theta_{2}: f_{2} \longrightarrow g_{2}: B \longrightarrow B^{\prime}
\end{aligned}
$$

using the notations $f_{1}, f_{2}, g_{1}, g_{2}$ given in (39). It is possible to define the Gray tensor product $\mathscr{A} \otimes \mathscr{B}$ directly from there, along an idea developed by Bourke and Gurski in [6]. Every small 2-category $\mathscr{A}$ comes equipped with an underlying category of objects and morphisms noted $|\mathscr{A}|$ and with an underlying set of objects noted $\|\mathscr{A}\|$. The category $|\mathscr{A}|$ may be seen as a locally discrete 2-category, while the set $\|\mathscr{A}\|$ 
may be seen as a discrete 2-category. As such, they come equipped with a pair of canonical 2-functors

$$
\|\mathscr{A}\| \stackrel{\text { inj }}{\longrightarrow}|\mathscr{A}| \stackrel{\text { inj }}{\longrightarrow} \mathscr{A}
$$

From this follows that there exists a 2-functor

$$
|\mathscr{A}| \square|\mathscr{B}| \longrightarrow|\mathscr{A}| \times|\mathscr{B}| \stackrel{i n j \times i n j}{\longrightarrow} \mathscr{A} \times \mathscr{B}
$$

where the funny tensor product $|\mathscr{A}| \square|\mathscr{B}|$ of the categories $|\mathscr{A}|$ and $|\mathscr{B}|$ is defined as the pushout of the diagram below:

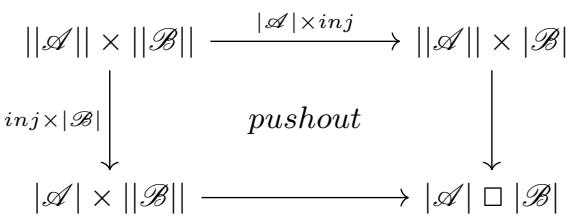

computed in the category Cat, see [6] for details. The Gray tensor product $\mathscr{A} \otimes \mathscr{B}$ is then characterized (or defined) as the unique 2-category such that the pair of 2-functors

$$
|\mathscr{A}| \square|\mathscr{B}| \stackrel{(a)}{\longrightarrow} \mathscr{A} \otimes \mathscr{B} \stackrel{(b)}{\longrightarrow} \mathscr{A} \times \mathscr{B}
$$

factors the composite 2-functor (40) in such a way that the 2-functor $(a)$ is an identity-on-objects and identity-onmorphisms 2-functor from $|\mathscr{A}| \square|\mathscr{B}|$ to $\mathscr{A} \otimes \mathscr{B}$ and the 2functor $(b)$ is a locally fully faithful 2-functor from $\mathscr{A} \otimes \mathscr{B}$ to $\mathscr{A} \times \mathscr{B}$. Note that the 2-functor $(b)$ coincides with the 2functor $q_{\mathscr{A}, \mathscr{B}}$ mentioned in $(38)$.

\section{B. Gray tensor product preserves coreflexive equalizers}

A pair of 2-functors

$$
\mathscr{A} \underset{G}{\stackrel{F}{\longrightarrow} \mathscr{B}}
$$

is called coreflexive when there exists a 2-functor $S: \mathscr{B} \rightarrow \mathscr{A}$ such that $S \circ F=S \circ G=\mathrm{id} \mathscr{A}$. An equalizer $\mathscr{E}$ of a coreflexive pair of 2-functors $F, G: \mathscr{A} \rightarrow \mathscr{B}$ in the category 2-Cat is called a coreflexive equalizer. Suppose that $\mathscr{E}$ is an equalizer of a coreflexive pair, as shown in the diagram below:

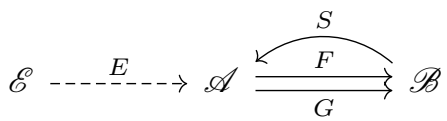

We establish that

Proposition 5: The Gray tensor product preserves coreflexive equalizers in the sense that for every 2-category $\mathscr{C}$, the diagram

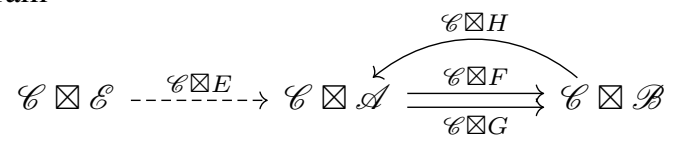

exhibits the 2-category $\mathscr{C} \otimes \mathscr{E}$ as a coreflexive equalizer of the 2-functors $\mathscr{C} \otimes F, \mathscr{C} \otimes G: \mathscr{C} \otimes \mathscr{A} \rightarrow \mathscr{C} \otimes \mathscr{B}$.

The fact is proved in three combined steps carefully described in the Appendix $C$.

\section{HOW EVERY ASYNCHRONOUS GRAPH CAN BE SEEN AS A 2-CATEGORY, FUNCTORIALLY}

We explain in this section how to define the functor

$$
\langle-\rangle: \text { Asynch } \longrightarrow \text { 2-Cat }
$$

which associates to every asynchronous graph $(G, \diamond)$ the 2 category $\langle G, \diamond\rangle$ whose objects and morphisms are the vertices and paths of the graph $G$, and whose 2-cells are reshufflings as defined in $\$ \mathrm{~V}-\mathrm{A}$. We finally observe in $\$ \sqrt{\mathrm{V}-\mathrm{B}}$ that there exists a coherent family of isomorphisms between 2-categories

$$
\left\langle G \amalg H, \diamond_{G} \omega H\right\rangle\left\langle G, \diamond_{G}\right\rangle \otimes\left\langle H, \diamond_{H}\right\rangle \quad\langle\mathbf{I}\rangle \cong \mathbf{1}
$$

where I denotes the neutral element of the shuffle tensor product of asynchronous graphs, and $\mathbf{1}$ denotes the terminal 2-category. This provides a firm conceptual foundation to the intuition that the Gray tensor product should be understood as an shuffle (or asynchronous) tensor product of 2-categories.

\section{A. Reshufflings between paths of an asynchronous graph}

Given an asynchronous graph $(G, \diamond)$, a reshuffling

$$
\varphi \quad: \quad f \longrightarrow g \quad: \quad x \longrightarrow y
$$

between two paths $f, g$ of the graph $G$ with same source $x$, same target $y$, and same length

$$
\begin{aligned}
f & =x \stackrel{u_{1}}{\longrightarrow} z_{1} \stackrel{u_{2}}{\longrightarrow} \cdots \stackrel{u_{k-1}}{\longrightarrow} z_{k-1} \stackrel{u_{k}}{\longrightarrow} y \\
g & =x \stackrel{v_{1}}{\longrightarrow} z_{1}^{\prime} \stackrel{v_{2}}{\longrightarrow} \cdots \stackrel{v_{k-1}}{\longrightarrow} z_{k-1}^{\prime} \stackrel{v_{k}}{\longrightarrow} y
\end{aligned}
$$

is defined as a bijection

$$
\varphi:\{1, \ldots, k\} \longrightarrow\{1, \ldots, k\}
$$

tracking a sequence of permutation tiles in $\diamond$ transforming the path $f$ into the path $g$. Typically, the permutation tile 35 is tracked by the reshuffling $\varphi:\{1,2\} \mapsto\{1,2\}$ defined as $1 \mapsto 2$ and $2 \mapsto 1$ and represented below using the two arrows:

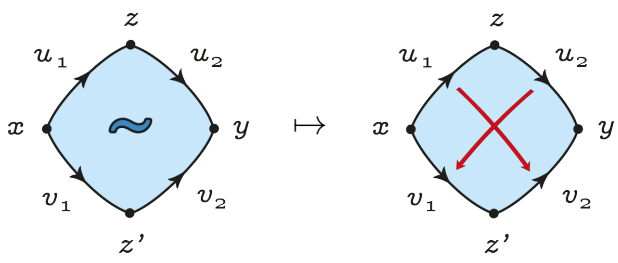

Similarly, the sequence of three permutations below is tracked by the reshuffling $\varphi:\{1,2,3\} \mapsto\{1,2,3\}$ defined as $1 \mapsto 3$, $2 \mapsto 3,3 \mapsto 1$, and represented using the three arrows:

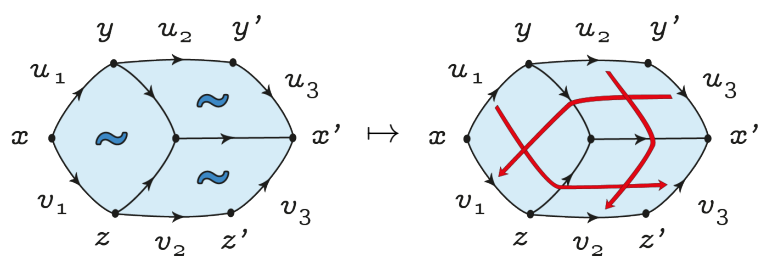


One main benefit of using the notion of reshuffling is that it enables to identify the two sequences of permutation tiles below as the very same reshuffling $\varphi:\{1,2,3\} \mapsto\{1,2,3\}$ :

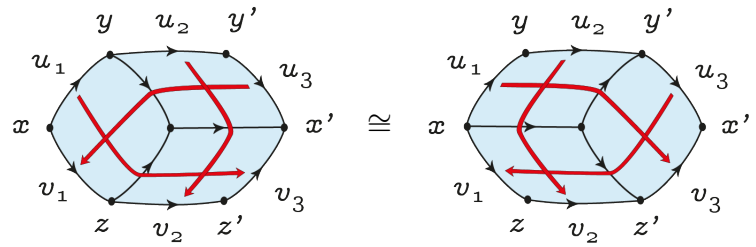

Similarly, the sequence of two inverse permutation tiles on the left-hand side is tracked by the identity reshuffling $\varphi$ : $\{1,2\} \rightarrow\{1,2\}$ and thus identified to the empty sequence of permutation tiles on the right-hand side:

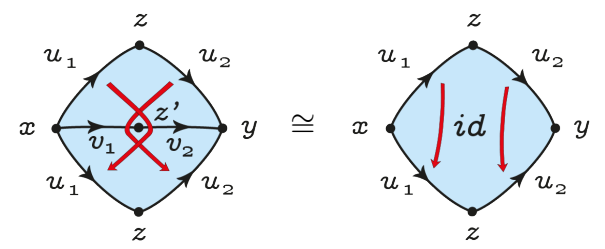

Although the notion of a reshuffling (42) tracking a sequence of permutation tiles in the asynchronous graph $(G, \diamond)$ should be intuitive at this stage, we give a detailed and explicit definition in the Appendix, see $\$ \mathrm{D}$.

At a more conceptual level, the 2-category $\langle G, \diamond\rangle$ may be characterized as the 2-category freely generated by the vertices and edges of the graph $G$ at the dimensions 0 and 1 , by the family of 2-cells

$$
\sigma_{f, g} \quad: \quad f \Longrightarrow g \quad: \quad x \longrightarrow y
$$

indexed by set of permutation tiles $f \diamond g$ in $(G, \diamond)$ at dimension 2, modulo the two families of equations between 2-cells depicted in the diagrammatic notations of 45 and (46).

\section{B. The symmetric monoidal functor}

Once the functor $\langle-\rangle$ explicated, it is not difficult to establish that it defines in fact a symmetric monoidal functor

$$
\langle-\rangle \quad: \quad(\text { Asynch }, \amalg, \mathbf{I}) \quad \longrightarrow \quad(2-C a t, \otimes, \mathbf{1})
$$

which preserves the shuffle tensor product up to isomorphisms

$$
\left\langle G \amalg H, \diamond_{G \amalg H}\right\rangle \cong\left\langle G, \diamond_{G}\right\rangle \otimes\left\langle H, \diamond_{H}\right\rangle \quad\langle\mathbf{I}\rangle \cong \mathbf{1}
$$

in the category 2-Cat. The existence and coherence laws of the isomorphisms may be deduced from the explicit definition in $\$ \mathrm{~V}-\mathrm{A}$ of the functor $\langle-\rangle$ combined with the description of the Gray tensor product in $\$ \mathrm{IV}-\mathrm{A}$. We also note that although the functor $\langle-\rangle$ does not preserve cartesian products, it does preserve equalizers.

\section{The WEAK DOUble CATEgory OF Bicomodules}

Given a monoidal category $(\mathbb{S}, \bigotimes, \mathbf{1})$ with coreflexive equalizers, we construct the weak double category $\operatorname{Comod}(\mathbb{S})$ of comonoids and bicomodules between them. In order to perform the construction, we make the assumption that the tensor product preserves coreflexive equalizers componentwise, in the sense that for every object $A$ of the category $\mathbb{S}$, the functors

$$
A \otimes-: \mathbb{S} \rightarrow \mathbb{S} \quad-\otimes A: \mathbb{S} \rightarrow \mathbb{S}
$$

preserve coreflexive equalizers. We have in mind the example of the 2-category $\mathbb{S}=\mathbf{2}$-Cat of small 2-categories, equipped with the Gray tensor product, see $\$$ IV-B. We define in $\$ \mathrm{VI}-\mathrm{A}$ and $\$ \mathrm{VI}-\mathrm{B}$ the vertical and horizontal maps of the weak double category $\operatorname{Comod}(\mathbb{S})$ and then describe its double cells in $\$ \mathrm{VI}-\mathrm{C}$

\section{A. Vertical maps in $\operatorname{Comod}(\mathbb{S})$}

The vertical category of $\operatorname{Comod}(\mathbb{S})$ is defined as the category Comonoid $(\mathbb{S}, \mathbb{Q}, \mathbf{1})$ of comonoids in the monoidal category $(\mathbb{S}, \mathbb{\bigotimes}, \mathbf{1})$. Recall that a comonoid $(A, d, e)$ is a triple consisting of an object $A$ of $\mathbb{S}$ and of a pair of morphisms

$$
d: A \longrightarrow A \otimes A \quad e: A \longrightarrow \mathbf{1}
$$

making the diagrams commute:
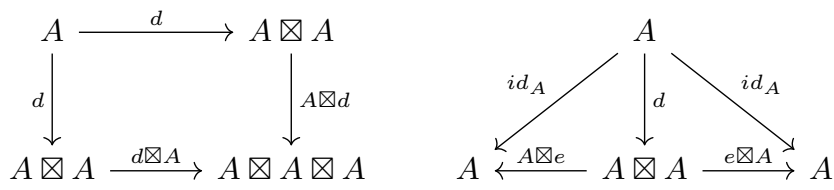

A comonoid homomorphism

$$
h:\left(A, d_{A}, e_{A}\right) \longrightarrow\left(B, d_{B}, e_{B}\right)
$$

is defined a morphism $h: A \rightarrow B$ making the two diagrams below commute:
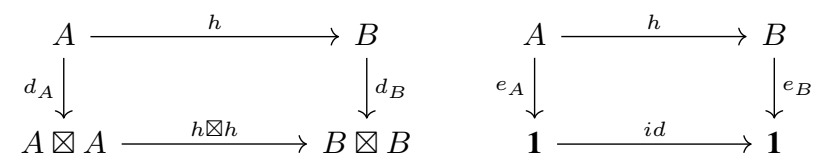

The category Comonoid $(\mathbb{S}, \bigotimes, \mathbf{1})$ is defined as the category whose objects are comonoids and whose morphisms are comonoid homomorphisms.

\section{B. Horizontal maps in $\operatorname{Comod}(\mathbb{S})$}

We recall the following definition.

Definition 4: An $A, B$-comodule

$$
S \quad: \quad A \stackrel{\longrightarrow}{\longrightarrow} B
$$

between two comonoids $\left(A, d_{A}, e_{A}\right)$ and $\left(B, d_{B}, e_{B}\right)$ is defined as an object $S$ equipped with a morphism

$$
\text { coact }_{S} \quad: \quad S \longrightarrow A \otimes S \otimes B
$$

such that the two diagrams commute

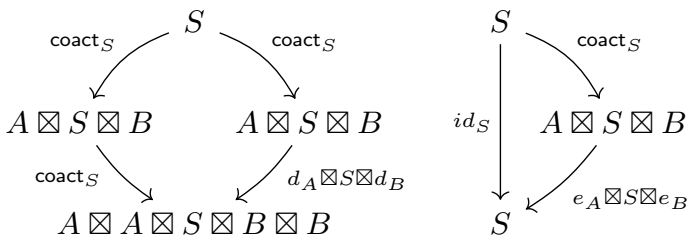


Given an $A, B$-comodule $S$, we use coact ${ }_{S}^{\text {left }}$ and $\operatorname{coact}_{S}^{\text {right }}$ as notations for the composite morphisms:

$$
\begin{gathered}
\text { coact }_{S}^{\text {left }}: S \stackrel{\text { coact }_{S}}{\longrightarrow} A \otimes S \otimes B \stackrel{A \otimes S \otimes e_{B}}{\longrightarrow} A \otimes S \\
\text { coact }_{S}^{\text {right }}: S \stackrel{\text { coact }_{S}}{\longrightarrow} A \otimes S \otimes B \stackrel{e_{A} \otimes S \otimes B}{\longrightarrow} S \otimes B
\end{gathered}
$$

which define a left comodule structure on the comonoid $A$ and a right comodule structure on the comonoid $B$, respectively. Given three comonoids $A, B$ and $C$, the composite of an $A, B$ comodule $S$ and of a $B, C$-comodule $T$

$$
S: A \longrightarrow B \quad T: B \longrightarrow C
$$

is the $A, C$-comodule

$$
S \otimes_{B} T: A \stackrel{\mid}{\longrightarrow} C
$$

defined as the equalizer of the coreflexive pair of morphisms coact $_{S}^{\text {right }} \otimes T$ and $S \otimes$ coact $_{T}^{\text {left }}$

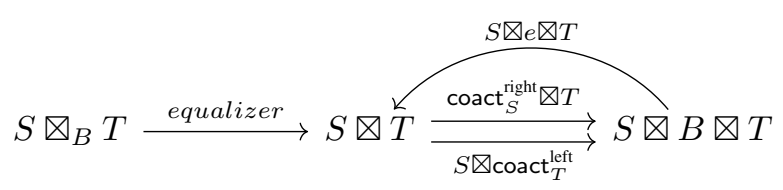

The $A, C$-comodule structure of $S \otimes_{B} T$ is given by the morphism coact $_{S \bigotimes_{B} T}$ defined as the unique solution of the universal problem represented below:

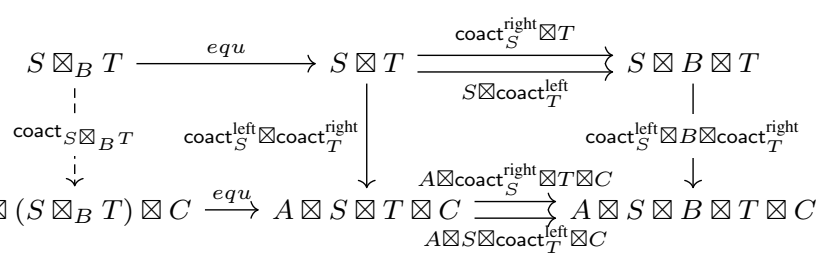

C. Double cells in $\operatorname{Comod}(\mathbb{S})$

A double cell in the double category $\operatorname{Comod}(\mathbb{S})$ of the form

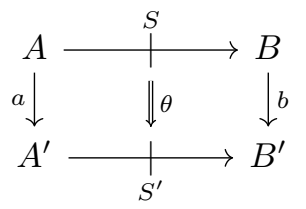

with horizontal edges an $A, B$-comodule $S$ and an $A^{\prime}, B^{\prime}$ comonoid $S^{\prime}$, and with vertical edges a pair of comonoid homomorphisms $a: A \rightarrow A^{\prime}$ and $b: B \rightarrow B^{\prime}$ is defined as a morphism

$$
\theta \quad: \quad S \longrightarrow S^{\prime}
$$

of the category $\mathbb{S}$ making the diagram below commute:

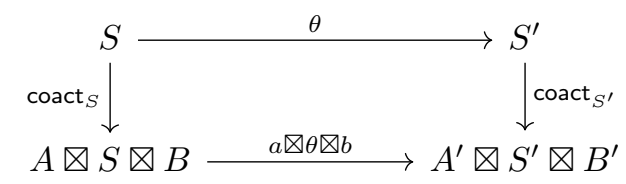

Given a pair of double cells

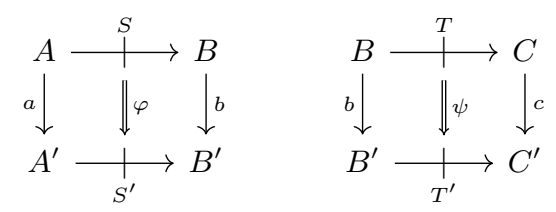

the composite double cell

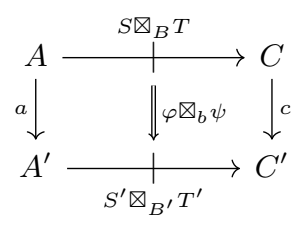

is defined as the unique morphism $\varphi \bigotimes_{b} \psi$ making the diagram below commute:

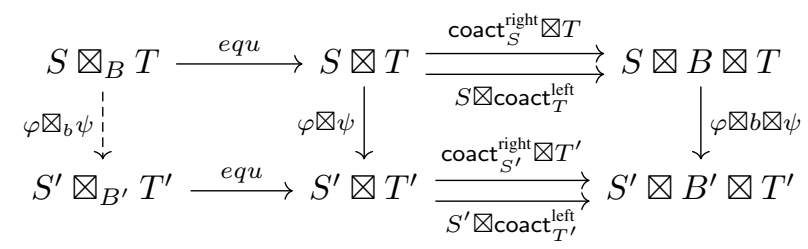

The following result is essentially folklore, especially when one sees the construction of $\operatorname{Comod}(\mathbb{S})$ as the dual of the construction of a weak double category of bimodules between monoids, see for instance [29].

Theorem 2: $\operatorname{Comod}(\mathbb{S})$ defines a weak double category.

Note that the assumption that the tensor product preserves coreflexive equalizers componentwise in $\mathbb{S}$ plays a critical role in the proof that horizontal composition is associative in $\operatorname{Comod}(\mathbb{S})$.

\section{THE TEMPLATE $t_{\text {asynch }}$ OF ASYNCHRONOUS GAMES}

In this section, we recall in $\$$ VII-A the notion of an internal category in a monoidal category $(\mathbb{S}, \bigotimes, \mathbf{1})$ as defined by Aguiar [3]. We then show that the template $t_{\text {asynch }}$ of asynchronous games formulated in the introduction defines an internal category in $(\mathbf{2}$-Cat, $\bigotimes, \mathbf{1})$.

\section{A. Definition of internal category in a monoidal category $\mathbb{S}$}

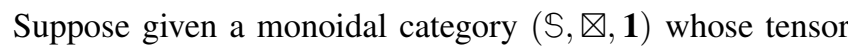
product preserves coreflexive equalizers componentwise, We start with the following definition:

Definition 5 (internal category): An internal category $t$ in the monoidal category $(\mathbb{S}, \mathbb{\otimes}, \mathbf{1})$ is defined as a monad in the weak double category $\operatorname{Comod}(\mathbb{S})$.

The definition may be expounded as follows: an internal category $t$ in $(\mathbb{S}, \mathbb{\otimes}, \mathbf{1})$ is a quadruple

$$
\star \quad=(\star[0], \star[1], \text { mult, unit })
$$

consisting of a comonoid $\star[0]$ with comultiplication and counit noted

$$
d: \star[0] \longrightarrow \star[0] \otimes \star[0] \quad e: \star[0] \longrightarrow \mathbf{1}
$$

together with an $\star[0], \star[0]$-bicomodule

$$
\star[1]: \quad \star[0] \longrightarrow+\downarrow[0]
$$

defined by a morphism in $\mathbb{S}$

$$
\text { coact }_{\star}: \star[1] \longrightarrow \star[0] \bigotimes \star[1] \bigotimes \star[1]
$$


making the diagrams (47) commute, together with double cells
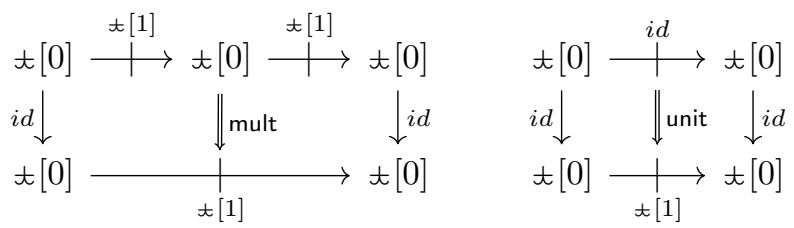

defined by a pair of morphisms in $\mathbb{S}$

$$
\begin{gathered}
\text { mult }: \star[1] \otimes_{\star[0]} \star[1] \longrightarrow \star[1] \\
\text { unit }: \star[0] \longrightarrow t[1]
\end{gathered}
$$

making the expected diagrams (49) commute. One requires moreover that mult and unit make the associativity and neutrality diagrams (53) and (53) commute as required of a monad $t$ in the weak double category $\operatorname{Comod}(\mathbb{S})$.

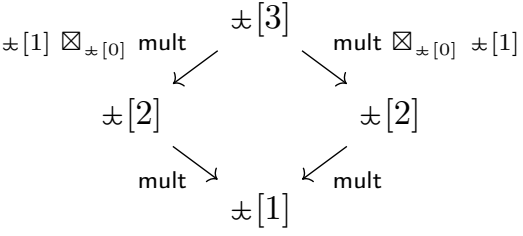

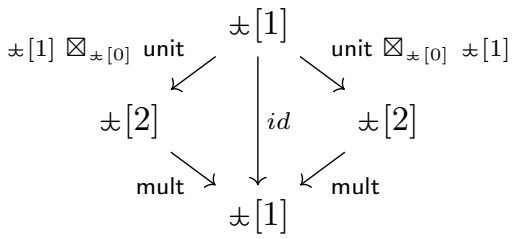

where we write

$$
\begin{array}{lcc}
\star[2] & = & \star[1] \bigotimes_{\star[0]} \star[1] \\
\star[3] & =\star[1] \bigotimes_{\star[0]} \star[1] \bigotimes_{\star[0]} \star[1]
\end{array}
$$

for the horizontal composites of the $\star[0], \star[0]-$ bicomodule (52) with itself.

\section{B. The template $t_{\text {asynch }}$ of asynchronous games}

We establish now that the template $t_{\text {asynch }}$ of asynchronous games defined in the introduction as the Gray comonoid

$$
\star_{\text {asynch }}[0]=\star\{\{\boldsymbol{O}, \boldsymbol{P}\}
$$

with comultiplication and counit (31) and the bicomodule

$$
\star_{\text {asynch }}[1]=\star\left\{\boldsymbol{O}_{s}, \boldsymbol{P}_{s}, \boldsymbol{O}_{t}, \boldsymbol{P}_{t}\right\}
$$

with coaction 32. The proof relies on the key observation that the horizontal composites $t_{\text {asynch }}[2]$ and $t_{\text {asynch }}[3]$ defined in (55) are themselves freely generated in the following way:

$$
\begin{gathered}
\star_{\text {asynch }}[2]=\star\left\{\boldsymbol{O}_{s}, \boldsymbol{P}_{s}, \boldsymbol{P O}, \boldsymbol{O P}, \boldsymbol{O}_{t}, \boldsymbol{P}_{t}\right\} \\
\star_{\text {asynch }}[3]=\star\left\{\left\{\boldsymbol{O}_{s}, \boldsymbol{P}_{s}, \boldsymbol{P} \boldsymbol{O}_{s}, \boldsymbol{O P}, \boldsymbol{P} \boldsymbol{O}_{t}, \boldsymbol{O P} \boldsymbol{P}_{t}, \boldsymbol{O}_{t}, \boldsymbol{P}_{t}\right\}\right.
\end{gathered}
$$

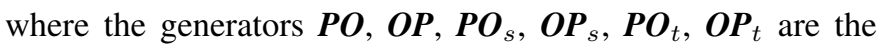
polarities of internal moves exchanged during the interaction between strategies. Note that we recover in this way a critical phenomenon and synchronization mechanism of traditional template games observed by Melliès [23].
Once this key observation has been made, the proof becomes a nice and enlightening combinatorial exercise, which essentially amounts to checking very carefully all the coherence diagrams required of an internal category in $\$ \mathrm{VII}-\mathrm{A}$ are indeed satisfied by $t_{\text {asynch }}$. At this stage, we observe that:

1. an asynchronous template game $\left(A, \lambda_{A}\right)$ as defined in Def. 1 is the same thing as an object of $\operatorname{Comod}(2-\operatorname{Cat}, \bigotimes, \mathbf{1})$ equipped with a vertical map $\lambda_{A}: A \longrightarrow t_{\text {asynch }}[0]$.

2. an asynchronous strategy $\sigma$ as defined in Def. 2 is the same thing as a double cell of the form

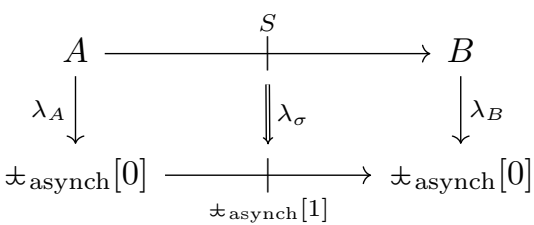

3. a notion of simulation

$$
\theta: \sigma \Longrightarrow \tau:\left(A, \lambda_{A}\right) \longrightarrow\left(B, \lambda_{B}\right)
$$

between asynchronous strategies $\sigma=\left(S, \lambda_{\sigma}\right)$ and $\tau=\left(T, \lambda_{\tau}\right)$ may be defined as a double cell in $\operatorname{Comod}(2-\operatorname{Cat}, \bigotimes, \mathbf{1})$

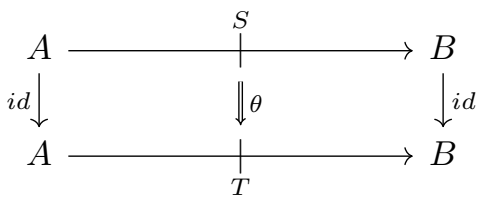

satisfying the expected property that $\lambda_{\sigma}$ concides with $\lambda_{\tau}$ vertically precomposed with the double cell $\theta$.

From these basic observations together with the property that $t_{\text {asynch }}$ defines an internal category in $(\mathbf{2}$-Cat, $\bigotimes, \mathbf{1})$, one easily deduces the main result of our paper, either directly or using the nice and general recipe in [11].

Theorem 3: Games $\left(t_{\text {asynch }}\right)$ defines a bicategory of asynchronous templates games, strategies and simulations.

\section{THE STAR-AUTONOMOUS BICATEGORY OF ASYNCHRONOUS TEMPLATE GAMES}

One main benefit of the structural approach to game semantics based on template games [23], [24] is that it becomes easier to establish in full precision and rigour that a given bicategory of games, strategies and simulations is symmetric monoidal closed, or even $*$-autonomous. This important aspect of template game semantics remains true in the asynchronous and 2-categorical framework developed in the present paper. Indeed, we establish that the bicategory Games $\left(t_{\text {asynch }}\right)$ of asynchonous template games is $*$-autonomous by observing

1. that the Gray tensor product $\otimes$ is symmetric and induces for that reason a tensor product on the double category $\operatorname{Comod}(2-C a t, \otimes, 1)$ which coincides with the tensor product of Gray comonoids on the vertical category. 
2. that the resulting Gray tensor product $\otimes$ induces in turn a tensor product $\nabla$ on the category of internal categories and internal functors in $(\mathbf{2}-\mathbf{C a t}, \bigotimes, \mathbf{1})$ where the notion of internal functor is defined as a monad morphism in $\operatorname{Comod}(2-C a t, \otimes, 1)$.

3. that the template $\star_{\text {asynch }}$ comes with an internal functor

$$
\otimes: \star_{\text {asynch }} \otimes t_{\text {asynch }} \longrightarrow t_{\text {asynch }}
$$

defined as the double cell in $\operatorname{Comod}(\mathbf{2 - C a t}, \bigotimes, \mathbf{1})$

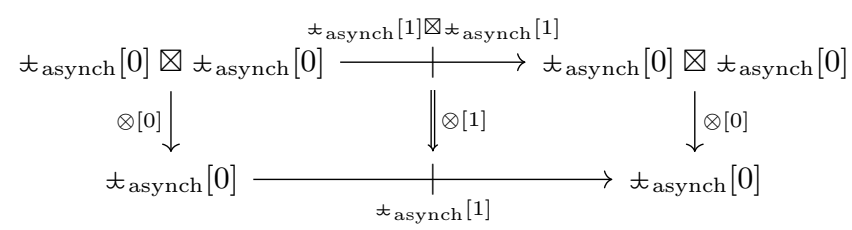

defined by the canonical Gray monoid structures $\otimes[0]$ and $\otimes[1]$ on $t_{\text {asynch }}[0]$ and $t_{\text {asynch }}[1]$ obtained by transporting the monoid structure $S \mapsto S+S$ of any set of generators along the symmetric monoidal functor $t\{-\}$ described in 30 .

Similarly, we observe that the template $t_{\text {asynch }}$ is equipped with an internal functor

$$
\text { neg : } \star_{\text {asynch }}^{o p} \longrightarrow t_{\text {asynch }}
$$

defined as the double cell in $\operatorname{Comod}(2-\operatorname{Cat}, \bigotimes, \mathbf{1})$

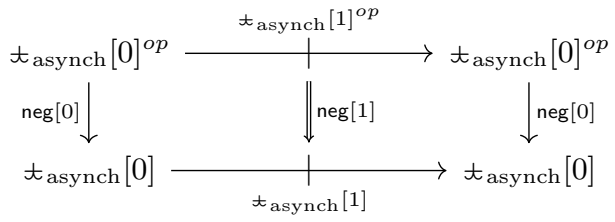

where the opposite $t^{o p}$ of an internal category is defined by permuting the outputs of the underlying comonoids and bicomodules using the symmetry sym of the Gray tensor product. Note that this purely algebraic operation in (2-Cat, $\bigotimes, \mathbf{1})$ reverses the left-to-right and right-to-left orientations of the template $t_{\text {asynch }}$, and thus the role of each polarity, as seen for instance in the comultiplication (31) discussed in the introduction:

$$
\star\{|\boldsymbol{O}, \boldsymbol{P}|\} \stackrel{d}{\longrightarrow} \star\{|\boldsymbol{O}, \boldsymbol{P}|\} \otimes \star\{\mid \boldsymbol{O}, \boldsymbol{P}\} \stackrel{\text { sym }}{\longrightarrow} \star\{|\boldsymbol{O}, \boldsymbol{P}|\} \otimes \star\{|\boldsymbol{O}, \boldsymbol{P}|\}
$$

We obtain in this way the main result of the paper:

Theorem 4: The bicategory Games $\left(t_{\text {asynch }}\right)$ is symmetric monoidal closed, and in fact $*$-autonomous.

\section{CONCLUSION AND FUTURE WORKS}

In this paper, we have shown how to upgrade to a properly asynchronous and 2-categorical framework the template game semantics designed by Melliès [23] for concurrent games. As we explain, the resulting concurrent game model based on the "asynchronous" Gray tensor product of 2-categories resolves a defect in the interpretation of deadlocks in the original semantics based on functorial spans and pullbacks of categories. One main challenge for future work will be to extend our current interpretation of multiplicative linear logic (MALL) to an asynchronous game semantics of differential linear logic, integrating to our 2-categorical setting the homotopy approach of [24]. We also believe that the formalism of asynchronous template games is sufficiently simple, general and conceptually clean to provide a unifying framework for various forms of sequential and concurrent game semantics.

\section{ACKNOWLEDGMENT}

The author would like to thank Pierre Clairambault for his early remarks on the treatment of deadlocks in the concurrent template game semantics, as well as the LICS reviewers for their helpful comments on the final version of the paper.

\section{REFERENCES}

[1] S. Abramsky, R. Jagadeesan, and P. Malacaria. Full abstraction for pcf. Information and Computation, 163(2):409-470, 2000.

[2] S. Abramsky and P.-A. Melliès. Concurrent games and full completeness. In Proceedings of the 14th IEEE Symposium on Logic in Computer Science (LICS), pages 431-442. IEEE Computer Society, 1999.

[3] M. Aguiar. Internal categories and quantum groups. $\mathrm{PhD}$ thesis, Cornell University, 1997.

[4] M. Batanin, D.-C. Cisinski, and M. Weber. Multitensor lifting and strictly unital higher category theory. Theory and Applications of Categories, 28(25):804-856, 2013.

[5] H. Bekič. Towards a mathematical theory of processes. Technical report tr 25.125, IBM Laboratory Vienna, 1971.

[6] J. Bourke and N. Gurski. The Gray tensor product via factorisation. Applied Categorical Structures, 25(4):603-624, August 2017.

[7] S. Castellan, P. Clairambault, S. Rideau, and G. Winskel. Games and strategies as event structures. Log. Methods Comput. Sci., 13(3), 2017.

[8] S. Castellan, P. Clairambault, and G. Winskel. Symmetry in asynchronous games. In Proceedings of the 25th EACSL Conference on Computer Science Logic and 29th ACM/IEEE Symposium Logic in Computer Science (CSL-LICS). EACSL and ACM/IEEE, 2014.

[9] S. Castellan, P. Clairambault, and G. Winskel. Thin Games with Symmetry and Concurrent Hyland-Ong Games. Logical Methods in Computer Science, Mar. 2019.

[10] C. Eberhart and T. Hirschowitz. What's in a game?: A theory of game models. In A. Dawar and E. Grädel, editors, Proceedings of the 33rd Annual ACM/IEEE Symposium on Logic in Computer Science, LICS 2018, Oxford, UK, July 09-12, 2018, pages 374-383. ACM, 2018.

[11] C. Eberhart, T. Hirschowitz, and A. Laouar. Template games, simple games, and day convolution. In Proceedings of Formal Structures of Computation and Deduction (FSCD), 2019.

[12] L. Fajstrup, E. Goubault, E. Haucourt, S. Mimram, and M. Raussen. Directed Algebraic Topology and Concurrency. Springer, 2016.

[13] R. Gordon, A. J. Power, and R. Street. Coherence for tricategories. Mem. Amer. Math Soc., 117, 1995.

[14] E. Goubault, T. Heindel, and S. Mimram. A geometric view of partial order reduction. Electronic Notes in Theoretical Computer Science, 298:179 - 195, 2013. Proceedings of the Twenty-ninth Conference on the Mathematical Foundations of Programming Semantics, MFPS XXIX.

[15] J. W. Gray. Formal Category Theory: Adjointness for 2-Categories. Number 391 in Lecture Notes in Mathematics. Springer Verlag, 1974.

[16] M. N. Gurski. An Algebraic Theory of Tricategories. University of Chicago, Department of Mathematics, 2006.

[17] N. Gurski. Coherence in Three-Dimensional Category Theory. Number 201 in Cambridge Tracts in Mathematics. Cambridge University Press, 2013.

[18] T. T. Hildebrandt and V. Sassone. Comparing transition systems with independence and asynchronous transition systems. In U. Montanari and V. Sassone, editors, CONCUR '96: Concurrency Theory, pages 84-97, Berlin, Heidelberg, 1996. Springer Berlin Heidelberg.

[19] M. Hyland and L. Ong. On full abstraction for pef. Information and Computation, 163(2):285-408, 2000.

[20] A. Mazurkiewicz. Concurrent program schemes and their interpretations. Daimi report pb 78, Aarhus University, 1977.

[21] P.-A. Melliès. Asynchronous games 3: An innocent model of linear logic. In Proceedings of the 10th Conference on Categorical Structures in Computer Science (CTCS 2004), volume 122, pages 171-192. Electronic Notes in Theoretical Computer Science, 2005. 
[22] P.-A. Melliès. Asynchronous games 2: the true concurrency of innocence. Theoretical Computer Science, 358(2-3):200-228, 2006.

[23] P.-A. Melliès. Categorical combinatorics of scheduling and synchronization in game semantics. Proceedings of the ACM on Programming Languages, 3 Issue POPL, January 2019.

[24] P.-A. Melliès. Template games and differential linear logic. In Proceedings of the 34th Annual ACM/IEEE Symposium on Logic in Computer Science (LICS), 2019.

[25] P.-A. Melliès and S. Mimram. Asynchronous games: Innocence without alternation. In 18th International Conference on Concurrency Theory (CONCUR 2007), pages 395-411, 2007.

[26] C. A. Petri. Kommunikation mit Automaten. Schriften des rheinischwestfälischen institutes für instrumentelle mathematik an der universität bonn, Darmstadt Technical University, 1962.

[27] C. A. Petri. Fundamentals of a theory of asynchronous information flow. In Proceedings of the First IFIP World Computer Congress, pages 386-390. North Holland, 1963.

[28] S. Rideau and G. Winskel. Asynchronous strategies. In Proceedings of the 26th Annual Symposium on Logic in Computer Science (LICS), pages 409-418. IEEE, 2011.

[29] R. Street. Quantum Groups: A Path to Current Algebra. Australian Mathematical Society Lecture Series. Cambridge University Press, 2007.

\section{APPENDiX A}

PROOF OF THE EXISTENCE OF FINITE LIMITS

IN THE CATEGORY ASYNCH OF ASYNCHRONOUS GRAPHS (\$III-C, PROP.4P)

We have established in $\$$ III-C that the category Asynch has finite products. A second and less immediate property is that the category Asynch has all equalizers, and thus all finite limits. Indeed, given a pair of homomorphisms between asynchronous graphs

$$
\left(G, \diamond_{G}\right) \stackrel{f}{\underset{g}{\rightleftarrows}}\left(H, \diamond_{H}\right)
$$

the equalizer $\left(E, \diamond_{E}\right)$ may be defined as follows: its vertices are the vertices $x$ of the graph $G$ such that $f(x)=g(x)$ and its edges are the edges $u: x \rightarrow y$ such that $f(u)=g(u)$. Note that $E$ may be seen as a subgraph of $G$. Then, a pair of paths $p, q: x \rightarrow y$ of length 2 in the graph $E$ defines a permutation tile $p \diamond_{E} q$ precisely when the two paths $p, q$ define such a permutation tile $p \diamond_{G} q$ in the asynchronous graph $\left(G, \diamond_{G}\right)$. We establish that defined in this way, $\left(E, \diamond_{E}\right)$ satisfies the properties required of an asynchronous graph, $E$ may be seen as a subgraph of $G$. Then, by definition, a pair of paths $p, q: M \rightarrow N$ of length 2 in the graph $E$ defines a permutation tile $p \diamond_{E} q$ precisely when the two paths $p, q$ define such a permutation tile $p \diamond_{G} q$ in the asynchronous graph $\left(G, \diamond_{G}\right)$. At this stage, the main difficulty is to show that the pair $\left(E, \diamond_{E}\right)$ just constructed satisfies the three properties required of an asynchronous graph. Symmetry and determinism of permutations are immediately deduced from the fact the properties are satisfied by $\left(G, \diamond_{G}\right)$, while the cube property of $\left(E, \diamond_{E}\right)$ follows easily from the following observation:

Proposition 6: Suppose given a pair of paths $p, q: M \rightarrow$ $N$ of length 2 defining a permutation tile $p \diamond_{G} q$ in the asynchronous graph $\left(G, \diamond_{G}\right)$. Suppose moreover that the path $p=u_{1} \cdot u_{2}: M \rightarrow N$ is a path of the subgraph $E$ of the graph $G$. In that case, the path $q=v_{1} \cdot v_{2}: M \rightarrow N$ is also a path of the subgraph $E$ and $p \diamond_{E} q$.
PROOF In order to prove the property, suppose that $f\left(u_{1}\right)=$ $g\left(u_{1}\right)$ and $f\left(u_{2}\right)=g\left(u_{2}\right)$. By the property of a homomorphism, we know that $f\left(u_{1}\right) \cdot f\left(u_{2}\right) \diamond_{H} f\left(v_{1}\right) \cdot f\left(v_{2}\right)$ and that $g\left(u_{1}\right) \cdot g\left(u_{2}\right) \diamond_{H} g\left(v_{1}\right) \cdot g\left(v_{2}\right)$. Since $f\left(u_{1}\right) \cdot f\left(u_{2}\right)=$ $g\left(u_{1}\right) \cdot g\left(u_{2}\right)$, it follows by determinism of permutation in $\left(H, \diamond_{H}\right)$ that $f\left(v_{1}\right) \cdot f\left(v_{2}\right)=g\left(v_{1}\right) \cdot g\left(v_{2}\right)$ and thus that $f\left(v_{1}\right)=g\left(v_{1}\right)$ and $f\left(v_{2}\right)=g\left(v_{2}\right)$. From this, we conclude that $v_{1}$ and $v_{2}$ are edges in the subgraph $E$ of the graph $G$ and we conclude that the path $q=v_{1} \cdot v_{2}$ is a path of $E$ which defines a permutation tile $p \diamond_{E} q$.

\section{APPENDIX B}

\section{AN ALGEBRAIC PRESENTATION OF THE GRAY TENSOR PRODUCT}

We suppose given a pair of small 2-categories $\mathscr{A}, \mathscr{B}$ and define their Gray tensor product as the 2-category $\mathscr{A} \otimes \mathscr{B}$ described by generators and relations, in the following way. The construction is somewhat part of the folklore and may be found for instance in [17].

Definition of the 0-cells. The objects of the 2-category $\mathscr{A} \otimes \mathscr{B}$ are the pairs $(A, B)$ consisting of an object $A$ of $\mathscr{A}$ and an object $B$ of $\mathscr{B}$.

Definition of the 1-cells by generators and relations. Its 1-cells are generated by two families of 1-cells

$$
(A, B) \stackrel{(a, B)}{\longrightarrow}\left(A^{\prime}, B\right) \quad(A, B) \stackrel{(A, b)}{\longrightarrow}\left(A, B^{\prime}\right)
$$

indexed by the 1-cells $a: A \rightarrow A^{\prime}$ of the 2-category $\mathscr{A}$ and by the 1-cells $b: B \rightarrow B^{\prime}$ of the 2-category $\mathscr{B}$, respectively. The 1 -cells of the freely generated category are then quotiented by the relations:

$$
\begin{gathered}
(a, B) *^{h}\left(a^{\prime}, B\right)=\left(a *^{h} a^{\prime}, B\right) \\
\left(i d_{A}^{h}, B\right)=i d_{(A, B)}^{h} \\
(A, b) *^{h}\left(A, b^{\prime}\right)=\left(A, b *^{h} b^{\prime}\right) \\
\left(A, i d_{B}^{h}\right)=i d_{(A, B)}^{h}
\end{gathered}
$$

which can be also described as the four equations:

$$
\begin{gathered}
(A, B) \stackrel{(a, B)}{\longrightarrow}\left(A^{\prime}, B\right) \stackrel{\left(a^{\prime}, B\right)}{=}\left(A^{\prime \prime}, B\right) \\
(A, B) \stackrel{\left(a^{\prime} *^{h} a, B\right)}{\longrightarrow}\left(A^{\prime \prime}, B\right) \\
(A, B) \stackrel{\left(i d_{A}^{h}, B\right)}{\longrightarrow}(A, B) \\
(A, B) \stackrel{i d_{(A, B)}^{h}}{\longrightarrow}(A, B) \\
(A, B) \stackrel{(A, b)}{\longrightarrow}\left(A, B^{\prime}\right) \stackrel{\left(A, b^{\prime}\right)}{\longrightarrow}\left(A, B^{\prime \prime}\right) \\
(A, B) \stackrel{\left(A, b^{\prime} *^{h} b\right)}{\longrightarrow}\left(A, B^{\prime \prime}\right) \\
(A, B) \stackrel{\left(A, i d_{B}^{h}\right)}{\longrightarrow}(A, B) \\
(A, B) \stackrel{i d_{(A, B)}^{h}}{\longrightarrow}(A, B)
\end{gathered}
$$


Definition of the 2-cells by generators and relations. Its 2-cells are generated by four families of generators: the two families of 2-cells

$(A, B)$

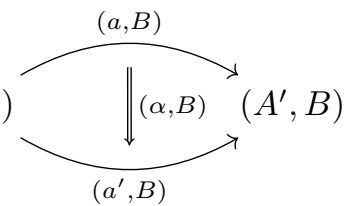

$(A, B)$

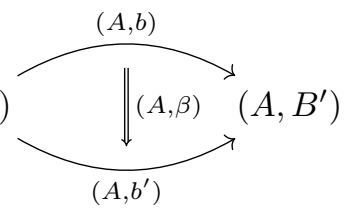

(58)

indexed by the 2-cells $\alpha: a \Rightarrow a^{\prime}: A \rightarrow A^{\prime}$ of the 2-category $\mathscr{A}$ and by the 2-cells $\beta: b \Rightarrow b^{\prime}: B \rightarrow B^{\prime}$ of the 2-category $\mathscr{B}$, respectively ; and the two families of 2-cells called Gray commutation:
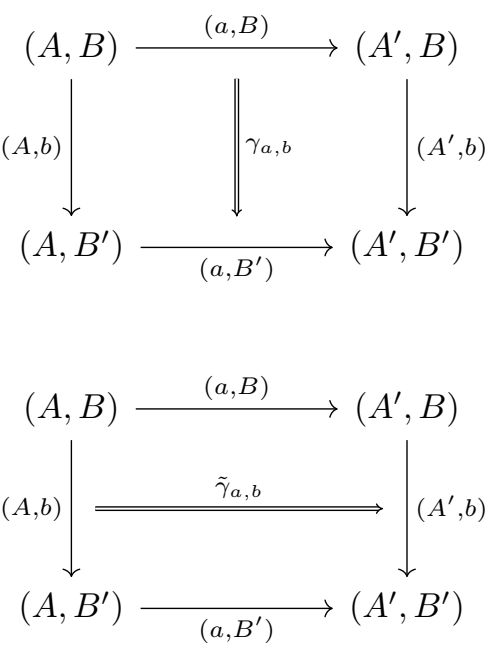

indexed by the 1-cells $a: A \rightarrow A^{\prime}$ of the 2-category $\mathscr{A}$ and the 1-cells $b: B \rightarrow B^{\prime}$ of the 2-category $\mathscr{B}$. The 2-cells of the 2-category freely generated by these generators are then quotiented by four families of relations. The first family of relations [a] regulates how the pairing interacts with vertical composition, while the three remaining families of relations [b-c-d] supervise the Gray commutation.

[a] Functoriality with respect to vertical composition: the first group of four relations enforces that the operations $b \mapsto$ $(A, b)$ and $a \mapsto(a, B)$ are functorial with respect to vertical composition, for every object $A$ of the 2-category $\mathscr{A}$ and every object $B$ of the 2-category $\mathscr{B}$ :

$$
\begin{gathered}
(\alpha, B) *^{v}\left(\alpha^{\prime}, B\right)=\left(\alpha *^{v} \alpha^{\prime}, B\right) \\
\left(i d_{a}^{v}, B\right)=i d_{(a, B)}^{v} \\
(A, \beta) *^{v}\left(A, \beta^{\prime}\right)=\left(\beta *^{v} \beta^{\prime}, B\right) \\
\left(A, i d_{b}^{v}\right)=i d_{(A, b)}^{v}
\end{gathered}
$$

The four equations between 2-cells are nicely depicted using pasting diagrams:
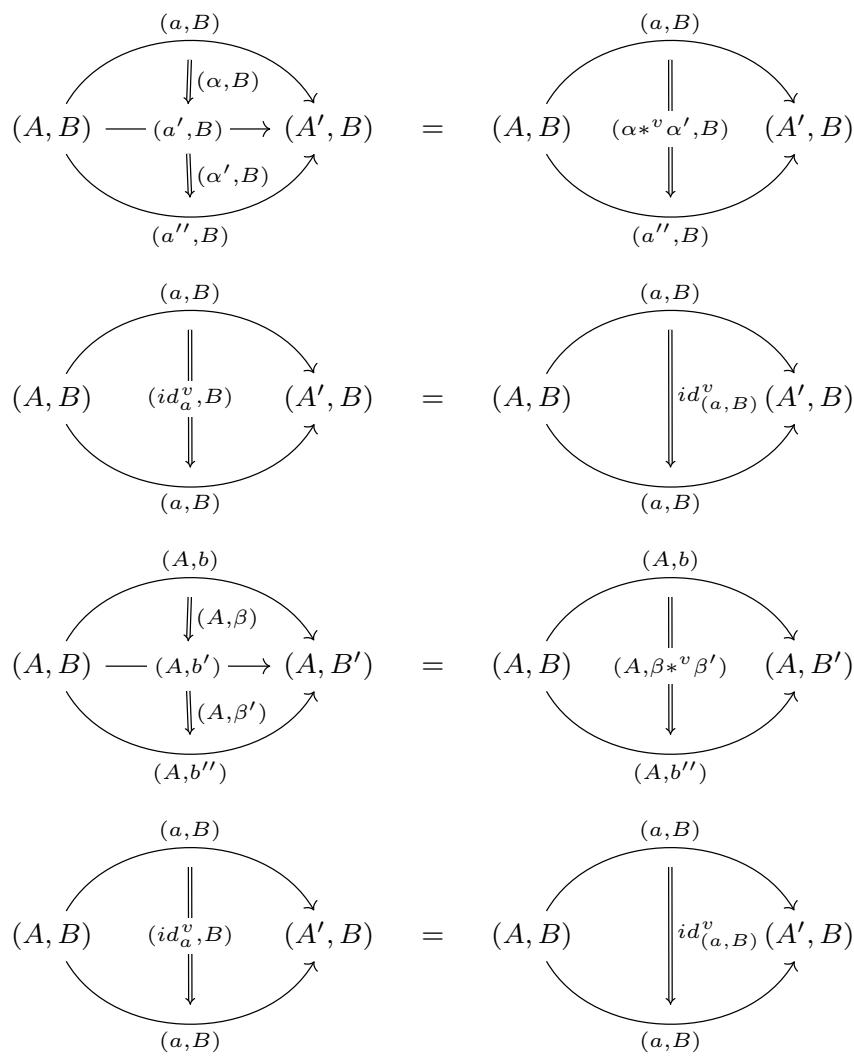

[b] Invertibility of the Gray commutation: the combination of two relations below indicates that the 2-cell $\gamma_{a, b}$ is invertible with the 2-cell $\tilde{\gamma}_{a, b}$ as vertical inverse:

$$
\begin{aligned}
& \gamma_{a, b} *^{v} \tilde{\gamma}_{a, b}=i d_{(a, B) *^{h}\left(A^{\prime}, b\right)}^{v} \\
& \tilde{\gamma}_{a, b} *^{v} \gamma_{a, b}=i d_{(A, b) *^{h}\left(a, B^{\prime}\right)}^{v}
\end{aligned}
$$

[c] Naturality of the Gray commutation: the group of four relations below enforces that the Gray commutation is natural with respect to vertical composition, both on the left and on the right side of the Gray tensor product:

$$
\begin{aligned}
& (\alpha, B) *^{v} \gamma_{a^{\prime}, b}=\gamma_{a, b} *^{v}\left(\alpha, B^{\prime}\right) \\
& \left(A^{\prime}, \beta\right) *^{v} \gamma_{a, b^{\prime}}=\gamma_{a, b} *^{v}(A, \beta)
\end{aligned}
$$

The two equations between 2-cells are nicely expressed in the diagrammatic language of pasting diagrams. The first equation says that the pasting diagram below

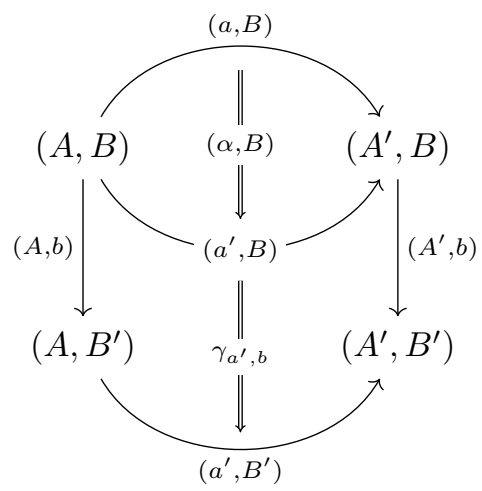


should be identified with the pasting diagram

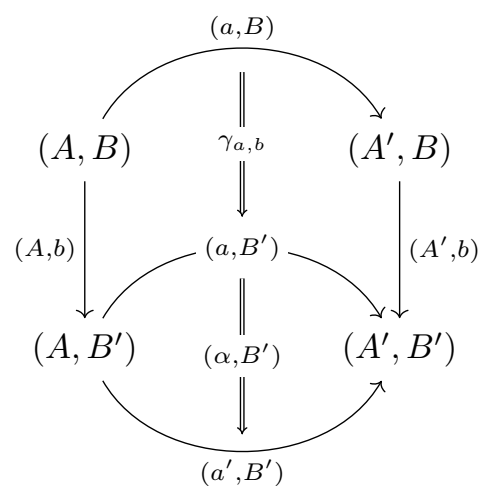

The second equation says that the pasting diagram below

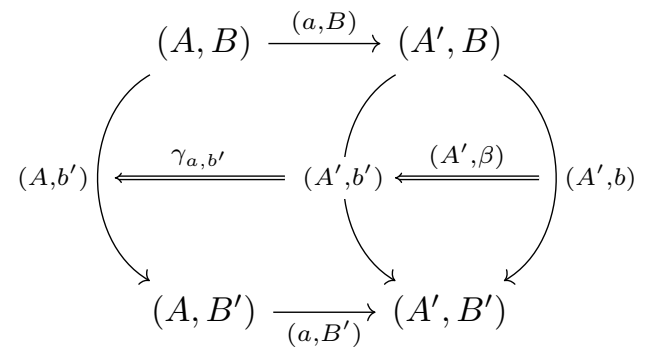

should be identified with the pasting diagram

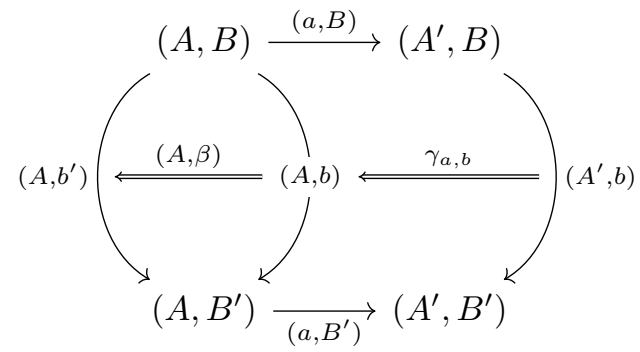

[d] Coherence of the Gray commutation: the group of four relations below should be understood as coherence properties of the Gray commutation, both on the left and on the right side of the Gray tensor product:

$$
\begin{gathered}
\left((a, B) *^{h} \gamma_{a^{\prime}, b}\right) *^{v}\left(\gamma_{a, b} *^{h}\left(a^{\prime}, B^{\prime}\right)\right)=\gamma_{a *^{h} a^{\prime}, b} \\
\gamma_{i d_{A}^{h}, b}=i d_{(A, b)}^{v} \\
\left(\gamma_{a, b} *^{h}\left(A^{\prime}, b^{\prime}\right)\right) *^{v}\left((A, b) *^{h} \gamma_{a, b}\right)=\gamma_{a, b *^{h} b^{\prime}} \\
\gamma_{a, i d_{B}^{h}}=i d_{(a, B)}^{v}
\end{gathered}
$$

The four equations between 2-cells are nicely expressed in the diagrammatic language of pasting diagrams. The first equation says that the pasting diagram

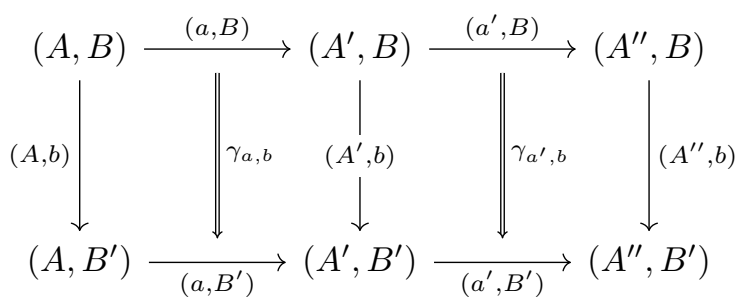

coincides with the 2-cell below:

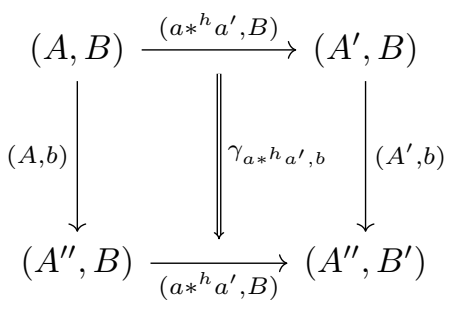

The second equation indicates that the 2-cell below

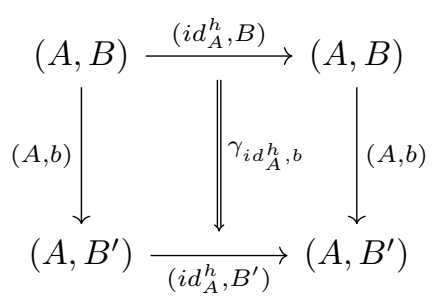

coincides with the identity 2 -cell

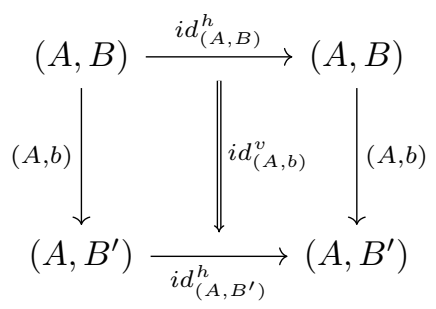

The third equation indicates that the pasting diagram

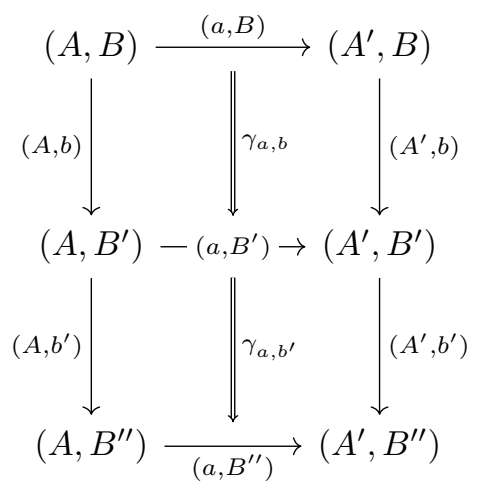

coincides with the 2-cell below:

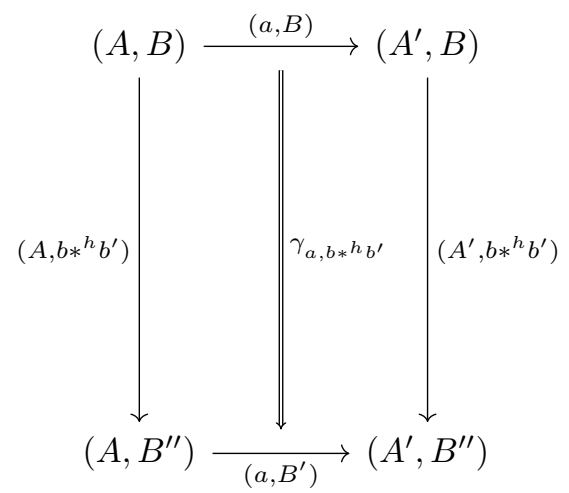


The fourth equation indicates that the 2-cell

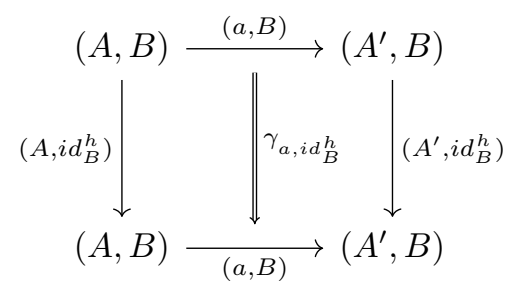

coincides with the identity 2-cell:

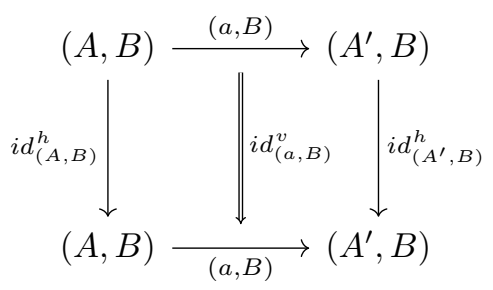

APPENDIX C

PROOF OF THE PRESERVATION OF COREFLEXIVE EQUALIZERS (PROP. 5 IN \$IV-B)

The preservation of coreflexive equalizers componentwise is proved in three combined steps. First, by universality of the cartesian product in 2-Cat, we know that the diagram

$$
\mathscr{C} \times \mathscr{E}-\mathscr{C} \times E \rightarrow \mathscr{C} \times \mathscr{A} \underset{\mathscr{C} \times G}{\stackrel{\mathscr{C} \times F}{\longrightarrow}} \mathscr{C} \times \mathscr{B}
$$

exhibits $\mathscr{C} \times \mathscr{E}$ as a coreflexive equalizer of the pair of 2functors $\mathscr{C} \times F$ and $\mathscr{C} \times G$. Then, we observe that the diagram

$$
|\mathscr{E}|-|E|-\rightarrow|\mathscr{A}| \stackrel{|F|}{\underset{|G|}{\longrightarrow}}|\mathscr{B}|
$$

is a coreflexive equalizer in Cat. A simple combinatorial exercise establishes that the functor

$$
\mathscr{D} \square-=\mathscr{X} \mapsto \mathscr{D} \square \mathscr{X} \quad: \quad \text { Cat } \longrightarrow \text { Cat }
$$

obtained by tensoring using the "funny tensor product" a category $\mathscr{X}$ with a fixed category $\mathscr{D}$ preserves coreflexive equalizers. From this follows that the diagram below

$$
|\mathscr{C}| \square|\mathscr{E}| \stackrel{|\mathscr{C}| \square|E|}{-}|\mathscr{C}| \square|\mathscr{A}| \stackrel{|\mathscr{C}| \square|F|}{\underset{|\mathscr{C}| \square|G|}{\longrightarrow}}|\mathscr{C}| \square|\mathscr{B}|
$$

exhibits the category $|\mathscr{C}| \square|\mathscr{E}|$ as the coreflexive equalizer in Cat of the pair of functors $|\mathscr{C}| \square|F|$ and $|\mathscr{C}| \square|G|$.

In the third and last step, we establish that (41) is an equalizer in 2-Cat by observing that given a 2-category $\mathscr{X}$, a 2-functor

$$
H: \mathscr{X} \rightarrow \mathscr{C} \otimes \mathscr{E}
$$

is the same thing as a pair $(\varphi, \psi)$ consisting of a functor

$$
\varphi:|\mathscr{X}| \rightarrow|\mathscr{C}| \square|\mathscr{E}|
$$

describing the 2-functor $H$ on objects and morphisms, and of a 2 -functor

$$
\psi: \mathscr{X} \rightarrow \mathscr{C} \times \mathscr{E}
$$

describing the 2-functor $H$ on cells, such that the diagram below

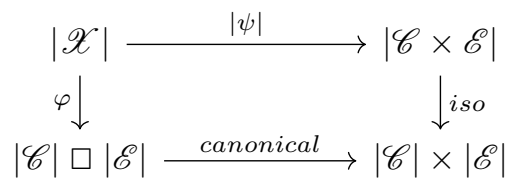

commutes in Cat. Using the fact that $|\mathscr{C}| \square|\mathscr{E}|$ and $\mathscr{C} \times \mathscr{E}$ are equalizers in Cat and 2-Cat, respectively, we conclude that it is the same thing as pair $\left(\varphi^{\prime}, \psi^{\prime}\right)$ consisting of a functor

$$
\varphi^{\prime}:|\mathscr{X}| \rightarrow|\mathscr{C}| \square|\mathscr{A}|
$$

and of a 2-functor

$$
\psi: \mathscr{X} \rightarrow \mathscr{C} \times \mathscr{A}
$$

such that

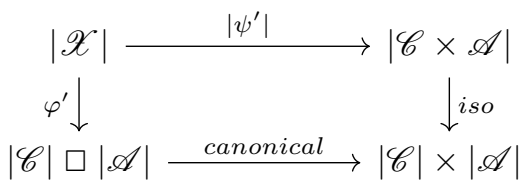

commutes, and making the expected diagrams commute between $F$ and $G$. We conclude that the Gray tensor product preserves coreflexive equalizers componentwise.

\section{APPENDIX D}

A DETAILED DESCRIPTION OF THE FUNCTOR FROM ASYNCHRONOUS GRAPHS TO 2-CATEGORIES

We give a detailed description of the functor

$$
\langle-\rangle:(\text { Asynch }, ш, \mathbf{I}) \longrightarrow(\text { 2-Cat }, \bigotimes, \mathbf{I})
$$

which associates to every asynchronous graph $(G, \diamond)$ a 2category $\langle G, \diamond\rangle$. As it is well-known, every graph $G$ induces a free category $\langle G\rangle$ whose objects are the vertices of $G$ and whose morphisms are the paths of $G$. In all this section, we suppose given an asynchronous graph $(G, \diamond)$ and describe how the construction $G \mapsto\langle G\rangle$ may be extended in order to associate a 2-category $\langle G, \diamond\rangle$ to the asynchronous graph $(G, \diamond)$. The starting point of the construction is as expected: the 2-category $\langle G, \diamond\rangle$ has the category $\langle G\rangle$ generated by $G$ as underlying category. Our main purpose in this section is thus to define the 2-cells of the 2-category $\langle G, \diamond\rangle$. This is done in two steps, as follows.

\section{A. From asynchronous graphs to sesquicategories}

A permutation step $\gamma=\left(h_{1}, p, q, h_{2}\right)$ is defined as a quadruple consisting of a pair of paths $p, q: P \rightarrow Q$ involved in a permutation tile $p \diamond q$ together with two paths $h_{1}: M \rightarrow P$ and $h_{2}: Q \rightarrow N$. We use the notation

$$
\gamma=h_{1} \cdot(p, q) \cdot h_{2} \quad: \quad f \longrightarrow g \quad: \quad x \longrightarrow y
$$

for such a permutation step $\gamma=\left(h_{1}, p, q, h_{2}\right)$, where $f, g$ : $x \rightarrow y$ are the two paths obtained by concatenation $f=h_{1}$. $p \cdot h_{2}$ and $g=h_{1} \cdot q \cdot h_{2}$ in the graph G. A permutation 
sequence $\varphi: f \Rightarrow g$ is defined as a sequence of permutation steps, of the following form:

$$
f=f_{1} \stackrel{\gamma_{1}}{\Longrightarrow} f_{2} \stackrel{\gamma_{2}}{\Longrightarrow} \cdots \stackrel{\gamma_{n}}{\Longrightarrow} f_{n+1}=g \quad: \quad x \rightarrow y
$$

Every pair of vertices $x, y$ of the asynchronous graph $(G, \diamond)$ induces a category noted $\operatorname{Perm}(x, y)$ whose objects are the paths $f: x \rightarrow y$ and whose morphisms are the permutation sequences $\varphi: f \Rightarrow g$. Composition in $\operatorname{Perm}(x, y)$ is called vertical composition and defined as concatenation of permutation sequences. The vertical composition of the permutation sequences

$$
\begin{aligned}
& \varphi: f \Longrightarrow g: x \longrightarrow y \\
& \psi \quad: \quad g \Longrightarrow h \quad: \quad x \longrightarrow y
\end{aligned}
$$

induces the permutation sequence noted

$$
\varphi *^{v} \psi \quad: \quad f \Longrightarrow h \quad: \quad x \longrightarrow y
$$

and defined by concatenation of $\varphi$ and $\psi$. It is worth observing that the category $\operatorname{Perm}(x, y)$ just defined is simply the free category generated by the permutation steps $\gamma: f \Rightarrow g$ between paths $f, g: x \rightarrow y$. The notation and terminology used for vertical composition is guided by the intuition that every permutation sequence $\varphi: f \Rightarrow g$ defines a 2-cell represented diagramatically as

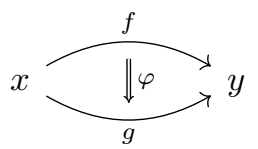

and that vertical composition corresponds to vertical diagram pasting:

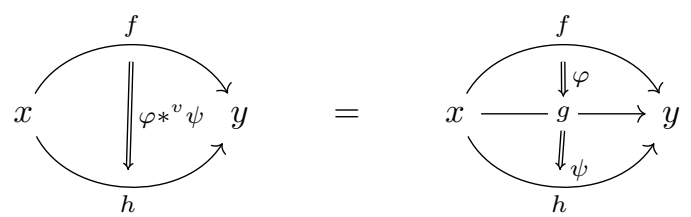

In order to establish the statement, we turn our attention to the definition of horizontal composition on paths and permutation sequences. The first thing to observe is that every permutation step of the form (66) can be extended by a pair of paths $j_{1}$ : $x^{\prime} \rightarrow x$ and $j_{2}: y \rightarrow y^{\prime}$ in order to obtain a permutation step

$$
j_{1} *^{h} \gamma *^{h} j_{2}: j_{1} \cdot f \cdot j_{2} \Longrightarrow j_{1} \cdot g \cdot j_{2}: x^{\prime} \rightarrow y^{\prime}
$$

defined as expected:

$$
j_{1} *^{h} \gamma *^{h} j_{2}=j_{1} \cdot h_{1} \cdot(p, q) \cdot h_{2} \cdot j_{2} .
$$

As we will see, there are good reasons for using the symbol $*^{h}$ as notation for this operation, which we call horizontal composition. In order to keep our notations consistent, we also allow ourselves to notation $*^{h}$ for concatenation of path, instead of the usual notation $p, q \mapsto p \cdot q$. In the same way as for permutation steps, every permutation sequence

$$
\varphi \quad: \quad f \longrightarrow g: x \longrightarrow y
$$

and every pair of paths $j_{1}: x^{\prime} \rightarrow x$ and $j_{2}: y \rightarrow y^{\prime}$ induce a permutation sequence

$$
\begin{aligned}
j_{1} *^{h} \varphi *^{h} j_{2} & : j_{1} *^{h} f *^{h} j_{2} \longrightarrow j_{1} *^{h} g *^{h} j_{2} \\
& : x^{\prime} \longrightarrow y^{\prime}
\end{aligned}
$$

obtained by extending every permutation step $\gamma_{i}$ defining the permutation sequence in (67) by horizontal composition with $j_{1}: x^{\prime} \rightarrow x$ and with $j_{2}: y \rightarrow y^{\prime}$, in the following way:

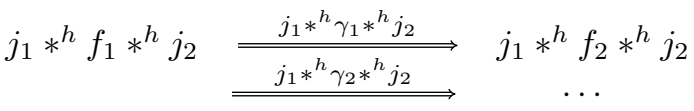

$$
\begin{aligned}
& \ldots \quad \stackrel{j_{1} *^{h} \gamma_{n} *^{h} j_{2}}{=} j_{1} *^{h} f_{n} *^{h} j_{2} \text {. }
\end{aligned}
$$

The notation and terminology we have just used for horizontal composition is justified by the idea that every permutation sequence $\varphi: f \Rightarrow g$ defines a 2 -cell

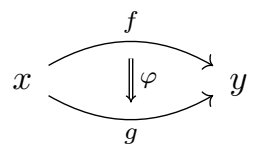

and that horizontal composition with $j_{1}: M^{\prime} \rightarrow M$ and $j_{2}$ : $N \rightarrow N^{\prime}$ defines a whiskering operation on the 2-cell, whose result may be depicted as follows:

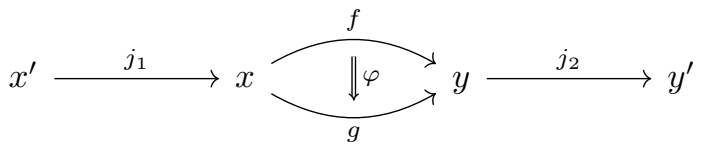

Before establishing that $\langle G, \diamond\rangle$ defines a 2-category (Prop. 7), we observe that the whiskering operation just defined satisfies all the properties expected of a sesquicategory. This means that

Proposition 7: Every asynchronous graph $(G, \diamond)$ defines a sesquicategory $\langle\langle G, \diamond\rangle\rangle$ with the vertices of $G$ as objects, the paths of $G$ as morphisms, the permutation sequences as 2-cells, and vertical composition $\varphi *^{v} \psi$ defined by concatenation of permutation sequences.

\section{B. From asynchronous graphs to 2-categories}

Coming back to the definition of permutation sequence, it is worth observing that every permutation step $\gamma: f \Rightarrow g$ : $M \rightarrow N$ relates two paths $f, g$ of the same length. From this follows that every path $f_{i}$ for $1 \leq i \leq n+1$ involved in the permutation sequence (67) has the same length, noted $k$. In particular, the two paths $f, g: M \rightarrow N$ have the same length, and thus both sets of indices $[f]$ and $[g]$ are equal to $\{1, \ldots, k\}$, see $\S ? ?$ for the notation. At this stage, we want to give a very simple recipe to associate a bijective function $[\varphi]:[f] \rightarrow[g]$ to a permutation sequence $\varphi: f \Rightarrow g$ of the form 67). Every permutation step $\gamma=\left(h_{1}, p, q, h_{2}\right)$ of the form (66) between two paths $f=u_{1} \cdots u_{k}$ and $g=v_{1} \cdots v_{k}$ of length $k$ defines a bijective function $[\gamma]:[f] \rightarrow[g]$ in the following way:

$$
[\gamma]=\left\{\begin{array}{l}
\ell+1 \mapsto \ell+2 \\
\ell+2 \mapsto \ell+1 \\
i \mapsto i
\end{array} \quad \text { when } i \leq \ell \text { or } i \geq \ell+3\right.
$$


where $\ell$ denotes the length of the path $h_{1}=u_{1} \cdots u_{\ell}=$ $v_{1} \cdots v_{\ell}$. The intuition behind the definition is that the bijective function

$$
[\gamma]:[f] \longrightarrow[g]
$$

reorders the edges of $f$ and $g$ in the way indicated by the permutation tile $p \diamond q$. Pictorially, the permutation step

$$
\gamma: f \longrightarrow g: x \longrightarrow y \text {. }
$$

with permutation tile $p \diamond q$ depicted below between $p=u_{\ell+1}$. $u_{\ell+2}$ and $q=v_{\ell+1} \cdot v_{\ell+2}$

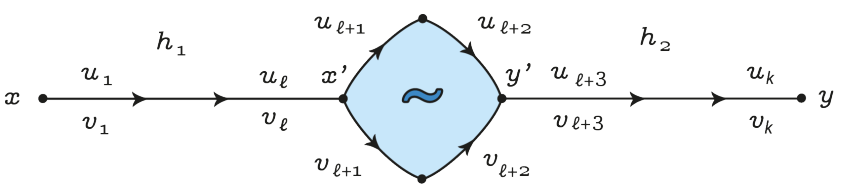

is turned into the bijective function $[\gamma]:[f] \rightarrow[g]$ on the set of indices $[f]=[g]=\{0, \ldots, k\}$ of edges appearing in the paths $f$ and $g$, defined as the transposition $\ell+1 \mapsto \ell+2$ and $\ell+2 \mapsto \ell+1$ indicated by the pair of purple (dark grey) arrows drawn on the permutation tile $p \diamond q$ :

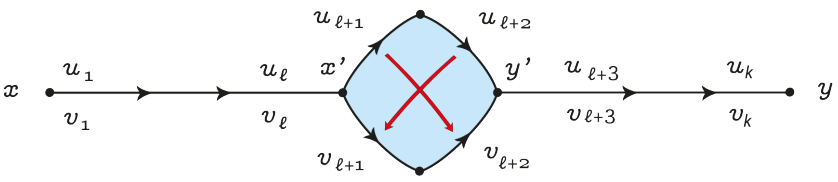

The definition just given of the bijective function

$$
[\gamma]:[f] \longrightarrow[g]
$$

associated to a permutation step

$$
\gamma: f \longrightarrow g: x \longrightarrow y \text {. }
$$

extends to a permutation sequence

$$
\varphi: f \longrightarrow g: x \longrightarrow y .
$$

in the expected way: the associated bijective function

$$
[\varphi]:[f] \longrightarrow[g]
$$

is defined as the (set-theoretic) composite of the bijective functions $\left[\gamma_{i}\right]:\left[f_{i}\right] \rightarrow\left[g_{i}\right]$ associated to each of the permutation steps $\gamma_{i}: f_{i} \Rightarrow f_{i+1}$, for $1 \leq i \leq k$. This definition can be simply expressed as the equation which says that the bijection

$$
[f] \stackrel{[\varphi]}{\longrightarrow}[g]
$$

is equal to the composite

$$
[f]=\left[f_{1}\right] \stackrel{\left[\gamma_{1}\right]}{\longrightarrow}\left[f_{2}\right] \stackrel{\left[\gamma_{2}\right]}{\longrightarrow} \cdots \stackrel{\left[\gamma_{n}\right]}{\longrightarrow}\left[f_{n+1}\right]=[g]
$$

This discussion leads us to introduce the equivalence relation $\cong$ which identifies two permutation sequences

$$
\varphi, \psi: f \longrightarrow g: x \longrightarrow y .
$$

precisely when they induce the same bijective function

$$
[\varphi]=[\psi]:[f] \longrightarrow[g]
$$

By way illustration, observe that the two permutation sequences

$$
\varphi, \psi: u_{1} \cdot u_{2} \cdot u_{3} \longrightarrow v_{1} \cdot v_{2} \cdot v_{3}: x \longrightarrow y
$$

involved in the cube property (36) induce the very same bijective function

$$
[\varphi],[\psi]:[f] \longrightarrow[g]
$$

which reverses the usual order on $\{1,2,3\}$ and thus turns every index $i \in\{1,2,3\}$ into the index $4-i \in\{1,2,3\}$. This means that the two permutations sequences $\varphi$ and $\psi$ are equivalent modulo $\cong$ in a situation nicely depicted as:
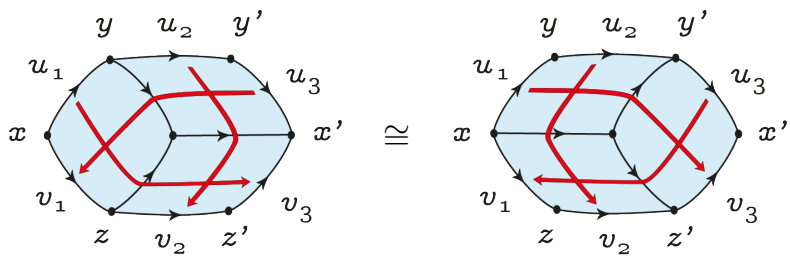

Suppose given two paths $f, g: x \rightarrow y$. We are now ready to define the notion of rescheduling of the form

$$
\varphi \quad: \quad f \longrightarrow g: x \longrightarrow y
$$

as a permutation sequence $\varphi: f \Rightarrow g$ considered modulo the equivalence relation $\cong$ just introduced. In other words, a rescheduling is an equivalence class modulo $\cong$ on the set of permutation sequences $\varphi: f \Rightarrow g$ transforming the path $f$ into the path $g$. We establish that:

Proposition 8: Every asynchronous graph $(G, \diamond)$ defines a 2-category $\langle G, \diamond\rangle$ with the vertices of $G$ as objects, the paths of $G$ as morphisms, and the reschedulings between paths as 2-cells. 


\section{APPENDIX E}

ASYNCHRONOUS STRATEGIES AS BICOMODULES

We find clarifying to illustrate with a few pictures the idea that an asynchronous strategy

$$
\sigma=\left(S, \operatorname{coact}_{\sigma}, \lambda_{\sigma}\right):\left(A, \lambda_{A}\right) \longrightarrow\left(B, \lambda_{B}\right)
$$

is described as a triple

$$
\left(S, \operatorname{coact}_{\sigma}, \lambda_{\sigma}\right)
$$

consisting of 2-category $S$ used as support, together with an $A, B$-bicomodule structure

$$
\operatorname{coact}_{\sigma} \quad: \quad S \longrightarrow A \otimes S \otimes B
$$

and a polarity 2 -functor

$$
\lambda_{\sigma} \quad: \quad S \longrightarrow \star_{\text {strat }}
$$

The coaction $\operatorname{coact}_{\sigma}$ may be represented diagrammatically as follows:

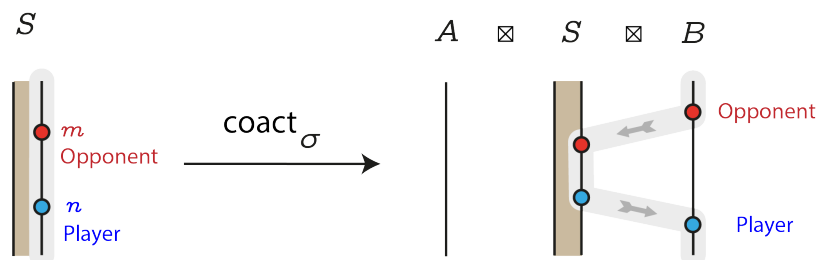

where the fact that the two moves $m$ and $n$ in the support $S$ are assigned the polarities

$$
\lambda_{\sigma} \quad: \quad m \mapsto \boldsymbol{O}_{t} \quad n \mapsto \boldsymbol{P}_{t}
$$

is indicated by their position on the brown (or grey) ribbon representing the strategy $\sigma$ in the picture. Similarly, the asynchronous strategy

$$
\tau=\left(T, \operatorname{coact}_{\tau}, \lambda_{\tau}\right):\left(B, \lambda_{B}\right) \longrightarrow\left(C, \lambda_{C}\right)
$$

is represented as

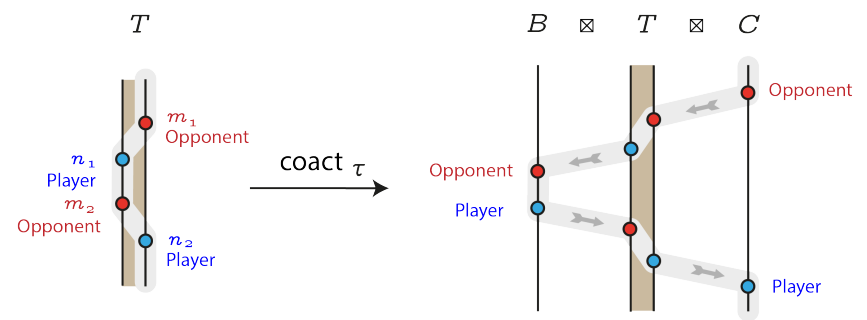

where the fact that the four moves $m_{1}, n_{1}, m_{2}$ and $n_{2}$ in the support $T$ are assigned the polarities

$\lambda_{\tau} \quad: \quad m_{1} \mapsto \boldsymbol{O}_{t} \quad n_{1} \mapsto \boldsymbol{P}_{s} \quad m_{2} \mapsto \boldsymbol{O}_{s} \quad n_{2} \mapsto \boldsymbol{P}_{t}$

is indicated by their position on the brown (or grey) ribbon representing the strategy $\tau$ in the picture. The composition of the two asynchronous strategies $\sigma$ and $\tau$ is then computed as the coreflexive equalizer

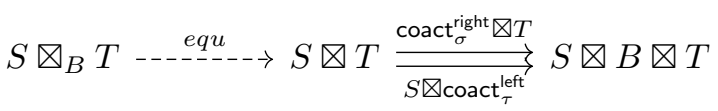

represented pictorially as follows:

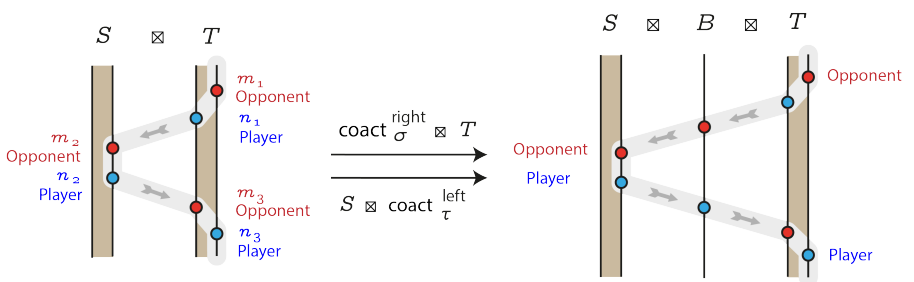

We indicate on the left a trajectory of six moves

$$
m_{1} \cdot n_{1} \cdot m_{2} \cdot n_{2} \cdot m_{3} \cdot n_{3}
$$

in the 2-category and template game $S \otimes T$ living at the same time in the coreflexive equalizer $S \otimes_{B} T$. Note that the two moves $n_{1}$ played by $\tau$ and $m_{2}$ played by $\sigma$ are equal moves when seen from the point of view of the game $B$. Similarly, the two moves $n_{2}$ played by $\sigma$ and $m_{3}$ played by $\tau$ are equal moves when seen from the point of view of the game $B$. This sequence of six moves may be thus described in $S \otimes_{B} T$ as a sequence of four moves:

$$
m_{1} \cdot m \cdot n \cdot n_{3}
$$

as depicted below

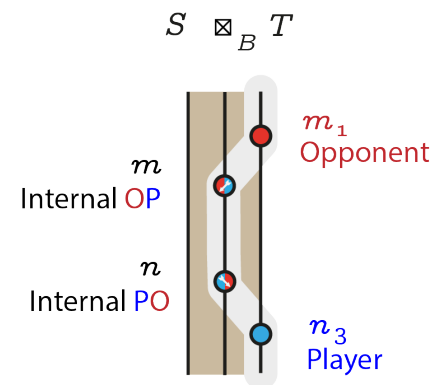

The position of the four moves on the brown (grey) area describing $S \otimes_{B} T$ indicate the polarity

$$
\begin{aligned}
& m_{1} \mapsto \boldsymbol{O}_{t} \quad m \mapsto \boldsymbol{P O} \\
& n \mapsto \boldsymbol{O P} \quad n_{3} \mapsto \boldsymbol{P}_{t}
\end{aligned}
$$

assigned in the template of polarities

$$
\left.\star_{\text {asynch }}[2]=\star\left\{\boldsymbol{O}_{s}, \boldsymbol{P}_{s}, \boldsymbol{P O}, \boldsymbol{O P}, \boldsymbol{O}_{t}, \boldsymbol{P}_{t}\right\}\right\}
$$

itself defined as the coreflexive equalizer

$$
\star_{\text {asynch }}[2]=\star[1] \otimes_{\star[0]} \star[1] .
$$

Note that postcomposing with multiplication

$$
\text { mult : } t_{\text {asynch }}[2] \longrightarrow t_{\text {asynch }}[1]
$$

gives rise to the polarity function

$$
\lambda_{\tau \circ \sigma}: S \otimes_{B} T \longrightarrow \star_{\text {asynch }}[2] \longrightarrow \star_{\text {asynch }}[1]
$$

on the four moves of the trajectory:

$$
\begin{aligned}
& m_{1} \mapsto \boldsymbol{O}_{t} \quad m \mapsto \operatorname{id}_{\langle *\rangle} \\
& n \mapsto \operatorname{id}_{\langle *\rangle} \quad n_{3} \mapsto \boldsymbol{P}_{t}
\end{aligned}
$$

in the template of polarities $\star_{\text {asynch }}[1]=\star_{\text {strat }}$. This polarity on the trajectory of the composite strategy $\tau \circ \sigma$ reflects the fact that the two moves $m$ and $n$ are considered as internal moves in the interaction between $\sigma$ and $\tau$; while the moves $m_{1}$ and $n_{3}$ of respective polarities $\boldsymbol{O}_{t}$ and $\boldsymbol{P}_{t}$ are the output of the interaction, played by $\tau \circ \sigma$ on the target game $C$. 